\title{
Deadwood volume in strictly protected, natural, and primeval forests in Poland
}

\author{
Leszek Bujoczek ${ }^{1}$ ( ) Janusz Szewczyk ${ }^{2} \cdot$ Małgorzata Bujoczek $^{2}$
}

Received: 29 November 2017 / Revised: 5 June 2018 / Accepted: 12 June 2018 / Published online: 28 June 2018

(c) The Author(s) 2018

\begin{abstract}
Standing and downed deadwood at different stages of decay provides a crucial habitat for a wide range of organisms. It is particularly abundant in unmanaged forests, such as strictly protected areas of national parks and nature reserves. The present work used the available data for such sites in Poland, analyzing a total of 113 studies concerning 79 sites to determine the causes contributing to variation in deadwood volume based on the duration of conservation, changes in deadwood volume over time (for those sites which were examined multiple times), elevation above sea level, forest type, stage of forest development, input of dead trees from the years preceding deadwood measurements, live tree volume, and the proportion of downed to standing deadwood). Depending on species composition and site altitude, most tree stands fell into one of four categories: subalpine spruce forests, montane beech-fir forests, low altitude beech-fir forests, or oak-hornbeam and riparian forests. The mean deadwood volume for all forest types amounted to $172.0 \mathrm{~m}^{3} / \mathrm{ha}$. The mean volume of deadwood in montane beech-fir forests $\left(223.9 \mathrm{~m}^{3} / \mathrm{ha}\right)$ was statistically significantly greater than in the other three forest types, for which it ranged from 103.5 to $142.5 \mathrm{~m}^{3} / \mathrm{ha}$. A direct effect of the duration of conservation on deadwood volume was not identified. Nevertheless, analysis of repeated measurements on the same sample plots at 10-year intervals showed a consistent rise in mean deadwood volume. A linear regression model for all the analyzed factors reported from montane beech-fir forests and subalpine spruce forests showed that in addition to site altitude, another statistically significant variable was the input of dead trees $\left(R^{2}=63.54 \%\right)$.
\end{abstract}

Keywords Old-growth forest $\cdot$ Volume $\cdot$ Coarse woody debris $\cdot$ Snags $\cdot$ Stumps $\cdot$ Biodiversity

\section{Introduction}

Surveys and monitoring of deadwood volume in forests provide useful indicators of habitat quality (Rondeux and Sanchez 2010). Previous research has shown that deadwood, whether standing or downed, constitutes an integral part of forest ecosystems, providing obligatory or facultative habitats for many organisms, such as bryophytes, lichens, fungi, and vascular plants (Dittrich et al. 2014; Preikša et al. 2015),

Communicated by Claus Bässler.

Leszek Bujoczek

leszek.bujoczek@ur.krakow.pl

1 Department of Forest Management, Geomatics and Forest Economics, Faculty of Forestry, University of Agriculture in Krakow, Al. 29 Listopada 46, Kraków 31-425, Poland

2 Department of Forest Biodiversity, Faculty of Forestry, University of Agriculture in Krakow, A1. 29 Listopada 46, Kraków 31-425, Poland as well as a variety of invertebrate and vertebrate animals (Bütler et al. 2004; Stokland et al. 2012). Of particular importance are nurse logs, which play a major role in the regeneration of tree stands under harsh climatic conditions (Zielonka 2006a). Deadwood is also a key factor in the nutrient cycle and a valuable carbon pool (Krankina and Harmon 1995; Merganičová and Merganič 2010).

Due to the significance of deadwood in contemporary forest management, efforts have been made to determine threshold values for biodiversity conservation. Review of data from European forests has revealed 36 thresholds ranging from 10 to $80 \mathrm{~m}^{3} /$ ha for boreal and lowland forests and from 10 to $150 \mathrm{~m}^{3} / \mathrm{ha}$ for mixed montane forest, with the peak values being $20-30 \mathrm{~m}^{3} / \mathrm{ha}$ for boreal forests, $30-40 \mathrm{~m}^{3}$ / ha for mixed montane forests, and $30-50 \mathrm{~m}^{3} /$ ha for lowland oak-beech forests (Müller and Bütler 2010). In addition to quantifying deadwood, it is also important to evaluate its degree of decay, species composition, and size distribution (heterogeneity of deadwood substrates) due to the diverse ecological requirements of saproxylic organisms (Stokland 
et al. 2012). Another crucial factor is the local climate of the site. In forest ecosystems, this is often linked to canopy openness, which determines the amount of light reaching the forest floor, modifying habitat conditions and affecting the species composition and population size of the organisms associated with deadwood (Seibold et al. 2016a, b). To ensure diversity of deadwood substrates and to foster natural dynamics in populations of species dependent on them, a constant supply of deadwood must be ensured over decades (Chećko et al. 2015).

One of the most effective ways of conserving endangered saproxylic species is the protection of their natural habitats by allowing dead trees to remain in the forest and gradually decompose (Gutowski 2006). This can be accomplished in strictly protected areas, and especially in sites from which deadwood has not been removed for decades. The number and size of such sites are affected by a range of biotic and abiotic factors, so differences among countries in this respect may be considerable.

In terms of both deadwood volume and other characteristics of tree stands (e.g. mean age and structure), managed forests differ significantly from natural and strictly protected ones (Bobiec 2002; Stachura-Skierczyńska and Bobiec 2008; WISL 2014). The forests of Poland constitute an important part of Central Europe's forests. The spatial distribution of different site conditions is largely reflected in the spatial structure of the dominant species. Except for montane areas, where Picea abies (L.) H. Karst., Abies alba Mill., and Fagus sylvatica L. are prevalent, in most tree stands across the country Pinus sylvestris L. is the most abundant species. According to data as of the end of 2013, the total forest area amounts to 9177.2 thousand ha (CSO 2014 ), or $29.4 \%$ of the country's area. As much as $81.2 \%$ of forests are state-owned, with $77.3 \%$ being managed by the State Forests National Forest Holding (SFNFH). In Poland the highest level of nature protection is afforded by 23 national parks occupying a total area of 314.6 thousand ha (individual parks ranging from 2.1 to 59.2 thousand ha). The average forest cover of national parks is $62 \%$, varying from 4 to $95 \%$ for individual parks (CSO 2014; Jamrozy 2014). The average age of forests managed by the SFNFH is 58 years with a stand volume of $272 \mathrm{~m}^{3} / \mathrm{ha}$, while the corresponding figures for national parks are 74 years and $348 \mathrm{~m}^{3} / \mathrm{ha}$. Tree stands which are 100 years old or older account for $24.1 \%$ of forests in national parks (WISL 2014). Another type of nature conservation in Poland encompasses 1480 reserves with different protection statuses (strict, active, landscape), which occupy a total area of 165.7 thousand ha (CSO 2014).

The mean volume of deadwood for all European forests, including data from the Russian Federation, is currently estimated at $20.5 \mathrm{~m}^{3} / \mathrm{ha}$, while without the Russian Federation it amounts to approximately $10 \mathrm{~m}^{3} / \mathrm{ha}$. Over the past two decades, deadwood volume has slightly increased in most regions of Europe. The amount of deadwood varies considerably depending on forest type, stand volume, rate of decay, and vegetation zone and is also influenced by forest management regimes (Van Brusselen 2011).

According to 2009-2013 data from more than 28,000 sample plots located all over Poland, the mean deadwood volume is $5.8 \mathrm{~m}^{3} / \mathrm{ha}$, with the figure for national parks (irrespective of their protection status) amounting to $36.7 \mathrm{~m}^{3} /$ ha (WISL 2014). Depending on the national park type, tree stand, and conservation objectives, the percentage share of strictly protected areas in different national parks ranges from 1 to $64 \%$. The total area subjected to strict protection in all national parks exceeds 712 thousand ha, or approximately $22 \%$ of their total area (Jamrozy 2014).

Deadwood in Polish forests has been systematically studied over the past years, leading to numerous reports on the subject. Some of them are part of wider research efforts, while others have not been released in electronic format or have not been published. However, to date no detailed overview of the existing, considerable body of data has been produced. Given that Polish forests occupy a large area in central Europe, such an overview would be interesting for scholars, practitioners, and environmental protection agencies. In view of the above, the objective of the present work was to:

- present comprehensive data on the volume of deadwood in natural and strictly protected forests;

- examine the relationship between deadwood volume and selected factors, such as site elevation a.s.l., forest type, live tree volume, duration of conservation, input of dead trees, and stage of forest development.

\section{Methodology}

\section{Data}

The review includes available data on deadwood volume in strictly protected forests ("Appendix"), some of which were reported as primeval. The term "forests of primeval character" was understood as forest complexes not affected by direct intervention of man (either entirely unharvested or only with individual trees removed-usually the largest ones), but possibly compromised by air pollution, road construction, etc. (Jaworski et al. 2002). The review includes data from many separate publications ("Appendix"), which vary depending on the methodology and detail of site description. Missing information has been either filled on the basis of other sources, or blank cells have been left. The reviewed data concern the volume of deadwood (standing and downed) as well as sampling methods, minimum diameters, live tree volume, elevation a.s.l., forest community characteristics, input of dead trees (the volume of trees that had died in the years 
preceding deadwood measurement), stage of forest development, measurement date, and the date when the national park was established or another form of conservation was instituted (subsequently operationalized as the "duration of conservation" variable). All the regions included in this paper are marked on the map in Fig. 1. The terminology used in this paper has the following meaning: a region is a national park, nature reserve, geographic region, etc., while a site is the exact place of study for which deadwood volume was reported for a given year. On some sites studies were carried out two or three times at intervals of up to more than a decade. In such cases, separate data are given for different years of measurement. The name of each site is provided in column 2 of "Appendix"; if several studies were conducted for a given site, the number of study is provided in parentheses. Finally, a study corresponds to an individual measurement, each presented as a separate line in "Appendix".

The data reported by individual studies (see "Appendix") were subsequently converted to standardized values for the purposes of the present work. The volumes have been standardized where the minimum diameter used to measure was $>5 \mathrm{~cm}$ diameter. The following regression was used: Volume $_{5 \mathrm{~cm}}=$ Volume $_{\mathrm{x} \mathrm{cm}} \cdot\left(0.0279 \times\right.$ diameter $\left._{\mathrm{x} \mathrm{cm}}+0.830\right)$ (Christensen et al. 2005).

\section{Duration of conservation}

For the purposes of this paper, duration of conservation was defined as the difference between the year when strict protection was instituted (usually provided by the authors of the reviewed papers) and the year in which deadwood measurement was conducted. If the initial year of conservation was not specified, the year of establishing the national park or reserve was used instead. Alternatively, duration of conservation was defined as the period over which deadwood had not been removed from the site, if such data were provided. On 29 sites measurements were repeated two or three times at 10 or 11-year intervals. For such sites, all available data were included ("Appendix").

\section{Forest elevation and forest type}

Most of the reviewed works specified site elevation a.s.l. If that information was provided as a range, an arithmetic mean was calculated. In the absence of such data, elevation was determined based on maps or other publications concerning a given site.

Forest type was determined on the basis of several criteria: elevation a.s.l., species composition, and plant community. The following four basic types were adopted:

Fig. 1 Regions in which sites for deadwood study were located

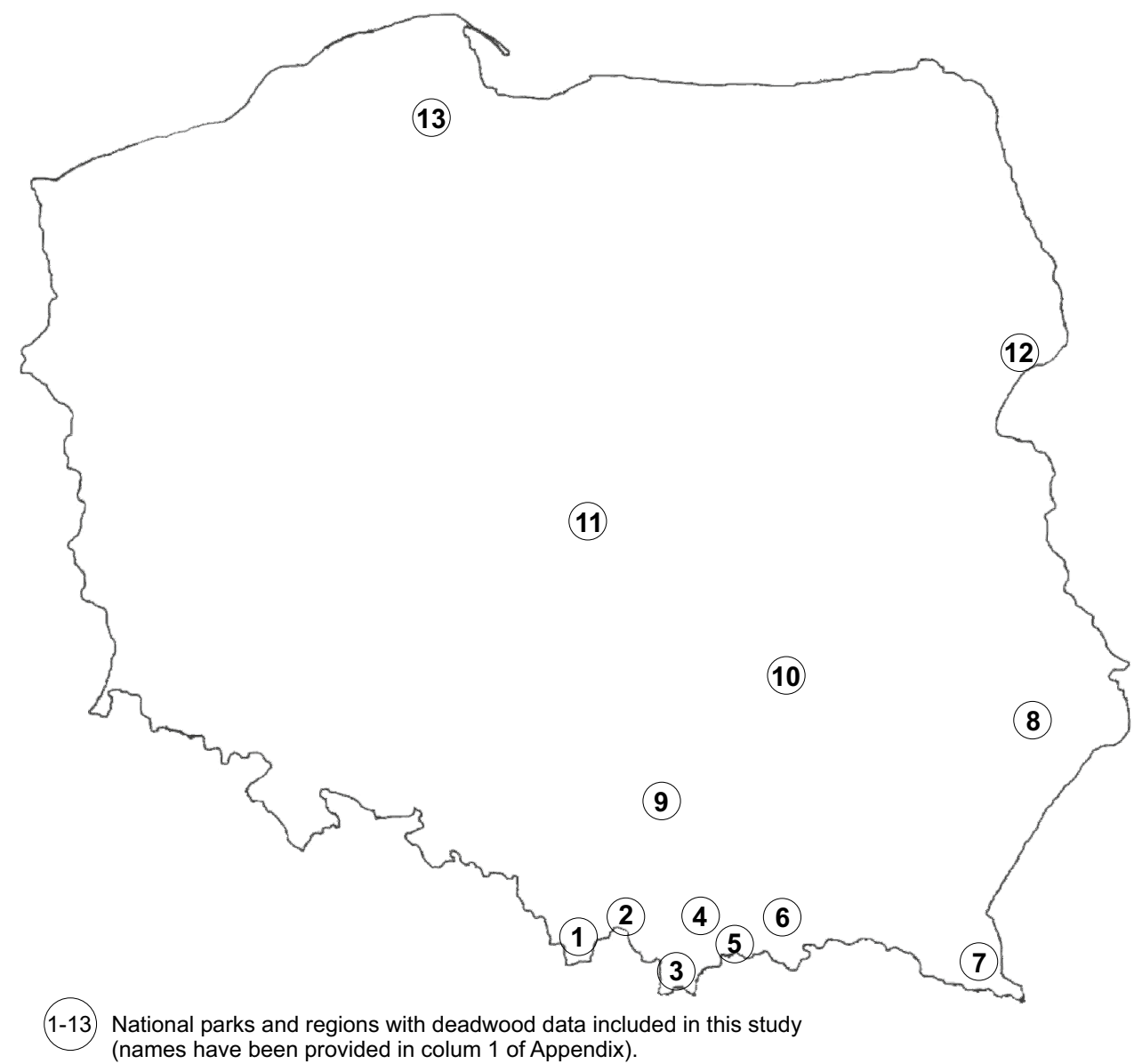


- Subalpine spruce forest-tree stands located above $1100 \mathrm{~m}$ a.s.l. with the typical community being Plagiothecio-Piceetum tatricum (Szaf., Pawł. et Kulcz. 1923) Br.-B1., Vlieg. et Siss. 1939 em. J. Mat. 1977 and with the dominant tree being Picea abies.

- Montane beech-fir forest-tree stands located from approximately 600 to $1150-1250 \mathrm{~m}$ a.s.l. (depending on the mountain range). The main communities include Dentario glandulosae-Fagetum W. Mat. 1964 ex Guzikowa et Kornaś 1969, Abietetum polonicum (Dziub. 1928) Br.-Bl. et Vlieg. 1939, and Carici-Fagetum abietetosum Pancer-Koteja 1973, Abieti-Piceetum montanum Szaf., Pawł. et Kulcz. 1923 em. J. Mat. 1978. The dominant trees are beech and fir, with some spruce and sycamore presence.

- Low altitude beech-fir forest-tree stands located below approximately $600 \mathrm{~m}$ a.s.1., with the typical communities being Abieto-Fagetum Kulczyński 1928, Dentario glandulosae Fagetum, Luzulo pilosae-Fagetum W. Mat. \& A. Mat. 1973 and Melico-Fagetum Lohm. ap Seibert 1954.

- Oak-hornbeam and riparian forests-tree stands belonging to the communities Tilio cordatae-Carpinetum betuli Tracz. 1962 and Circaeo-Alnetum Oberd. 1953.

Only two sampling sites were located in pine-oak forests with Querco roboris-Pinetum fagetosum (W. Mat. 1981) J. Mat. 1988 communities, and so they were excluded from analysis of deadwood volume per forest type. Some studies encompassed larger and more phytosociologically varied areas; they were not assigned to any of the above-mentioned types, either.

\section{Stage of forest development and input of dead trees}

The stage of forest development was determined based on the classification proposed by Korpel $(1989,1995)$ for primeval forests, taking into account their structure and growth dynamics. It should be noted that the duration and trajectory of the three stages identified (growing up, optimum, and breakup) depended on site conditions and species composition.

The other factor describing changes in forest stands was the volume of trees which had died in the period immediately preceding deadwood measurements on site, which reflects the input of dead trees to the ecosystem. This premeasurement period ranged from 5 to 21 years, and in the vast majority of cases amounted to 10 or 11 years. However, such data were available only for some of the sites, mostly for subalpine spruce forests and montane beech-fir forests. "Appendix" provides both the volume and period of time specified by the authors; the values used in the present analysis were converted into volume per decade.

\section{Data analysis}

Analysis of data concerning deadwood volume was carried out in two steps:

- selected individual factors (type of forest, elevation above sea level, share of standing deadwood in total deadwood volume, total deadwood to live tree volume ratio, duration of conservation) were studied to show present-day differences among strictly protected Polish forests;

- multiple regression analysis was used to identify those factors which have the greatest influence on deadwood volume variation in the studied sites.

The relationships between independent variables and deadwood volume were analyzed using different datasets, depending on the variable. The current values of the variables were taken from the latest studies from sites. Given that not all studies contained all the needed information, the number of data used for determining: total deadwood volume; live tree volume, deadwood volume in the various forest types, volume of downed deadwood, and volume of standing deadwood, ranged from 72 to 79 . All available data were used to determine relationships between these variables, and in particular between: total deadwood volume and site elevation a.s.l; duration of conservation and total deadwood volume; combined volume of deadwood and live trees; deadwood to live tree volume ratio; and standing deadwood to total deadwood volume ratio. The number of data used in these analyses ranged from 101 to 110 ; the differences were again due to certain data missing from some studies.

In terms of the number of total deadwood measurements per site (which was also subjected to statistical analysis), among the total of 77 sites for which data were reported, 48 were investigated once, 25 twice, and 4 three times. This has resulted in an additional 33 data for the 29 sites with multiple measurements. These data are expressed as differences with respect to previous measurements and presented in a separate figure to illustrate temporal changes in deadwood volume.

Analysis of variance, Student's $t$ test, Friedman's ANOVA and the Kruskal-Wallis test were used to determine the significance of differences. Relationships between variables were tested by means of Pearson's correlation coefficient and nonlinear regression. The above tests were conducted using STATISTICA 13 software.

In multiple regression analysis, models were constructed exclusively based on data from the latest measurements for the studied sites. Due to the absence of data on the input of dead trees in two forest types (low altitude beech-fir forest, oak-hornbeam and riparian forests) and the similarity of the variables "elevation a.s.l" and "forest type" (in this paper forest type is largely associated with site altitude), several different models were considered. The other variables, that 
is, live tree volume, duration of conservation, and stage of forest development, were present in all the analyzed models. Scatter plots were used to determine the dependence curves between the variables. The relationship between deadwood volume and elevation a.s.l. was a quadratic function.

Since the number of variables differed and models could not be estimated from the same data set, leave-one-out cross-validation was used, followed by calculation of the root mean squared error (RMSE):

$\mathrm{RMSE}=\sqrt{\frac{1}{n} \sum_{i=1}^{n} e_{i}^{2}}$

where $e_{i}$ is the error for $i$ th observation and $n$-the number of observations. Models with smaller RMSEs, with the best fit to data, were used in further analysis. Normal distribution of residuals and homoscedasticity of variances were analyzed using the Shapiro-Wilk and Breusch-Pagan tests. Calculations were made using R v. 3.4.3 software (R Core Team 2017).

\section{Results}

The mean deadwood volume was $172.0 \mathrm{~m}^{3} / \mathrm{ha}\left(\mathrm{SD}=101.3 \mathrm{~m}^{3} /\right.$ ha), with values for individual sites from 0 to $427 \mathrm{~m}^{3} / \mathrm{ha}$. The mean live tree volume on those sites was $526.4 \mathrm{~m}^{3} / \mathrm{ha}$ $\left(\mathrm{SD}=138 \mathrm{~m}^{3} / \mathrm{ha}\right.$ ), ranging from 211 to $907 \mathrm{~m}^{3} / \mathrm{ha}$.

The deadwood to live tree volume ratio was from 0 to $172 \%$ (Fig. 2). In two cases, deadwood volume exceeded live tree volume. The average ratio was $34.0 \%(\mathrm{SD}=24.5 \%)$. The combined volume of deadwood and live trees was from 270 to $1093 \mathrm{~m}^{3} / \mathrm{ha}$, with a mean value of $701 \mathrm{~m}^{3} / \mathrm{ha}$
$\left(\mathrm{SD}=171 \mathrm{~m}^{3} / \mathrm{ha}\right)$. The deadwood to combined volume of deadwood and live trees ratio was from 0 to $29.9 \%$ (Fig. 3). The average ratio was $5.0 \%(\mathrm{SD}=3.9 \%)$.

The duration of tree stand conservation was not significantly correlated with deadwood volume (Pearson's $r=0.14, p>0.05)$, but significant differences were found for repeated (two or three) measurements of the same sites at $10-11$ year intervals (Student's $t$ test $=3.74, p<0.001$ for double measurements; Chi-square ANOVA $=6.50, p<0.05$ for triple measurements). Deadwood volume increased considerably over the years, on average by slightly more than $30 \mathrm{~m}^{3} / \mathrm{ha} / \mathrm{decade}$ between first and second measurements and by $57 \mathrm{~m}^{3} / \mathrm{ha} /$ decade between the second and third measurements (Fig. 4). However, not all reports revealed an increase in deadwood volume after 10-11 years. Out of 33 measurements (Fig. 5), an increment was noted in 27 cases (by $0.4-155 \mathrm{~m}^{3} / \mathrm{ha} /$ decade), and a decline in 6 cases (by $1-31 \mathrm{~m}^{3} /$ ha/decade).

Deadwood volume varied considerably throughout the entire range of elevations a.s.l., with rather low values observed at all elevations (approximately $50 \mathrm{~m}^{3} / \mathrm{ha}$ ). The highest deadwood volumes were found for the 600-1000 $\mathrm{m}$ a.s.l. range. The relationship was statistically signiicant (quadratic regression analysis, $p<0.05$; Fig. 6). Also a comparison of sites by forest type (a variable associated with elevation) showed significant differences between deadwood volume in montane beech-fir forests $\left(223.9 \mathrm{~m}^{3} / \mathrm{ha}\right)$ and other forest types (ANOVA $F=7.99, p<0.001$ ). In the remaining three forest types, deadwood volume ranged from 103.5 to $142.5 \mathrm{~m}^{3} /$ ha (Fig. 7).

The mean volume of downed deadwood was $105.8 \mathrm{~m}^{3} /$ ha $\left(\mathrm{SD}=68.9 \mathrm{~m}^{3} / \mathrm{ha}\right)$ and that of standing deadwood was
Fig. 2 Relationship between live tree volume and deadwood volume for the various studies (the dashed horizontal line indicates the mean total deadwood/live tree volume ratio (\%), which is $34.0 \%$ )

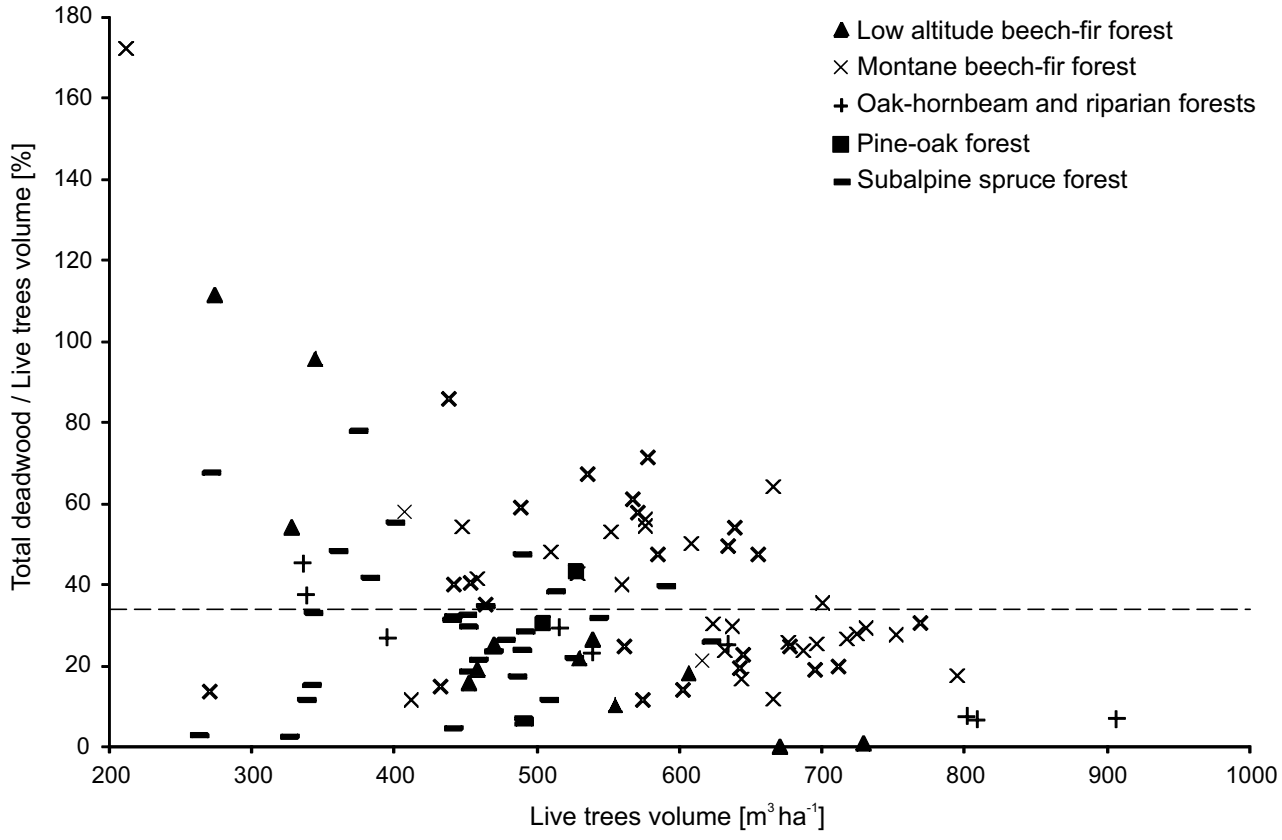




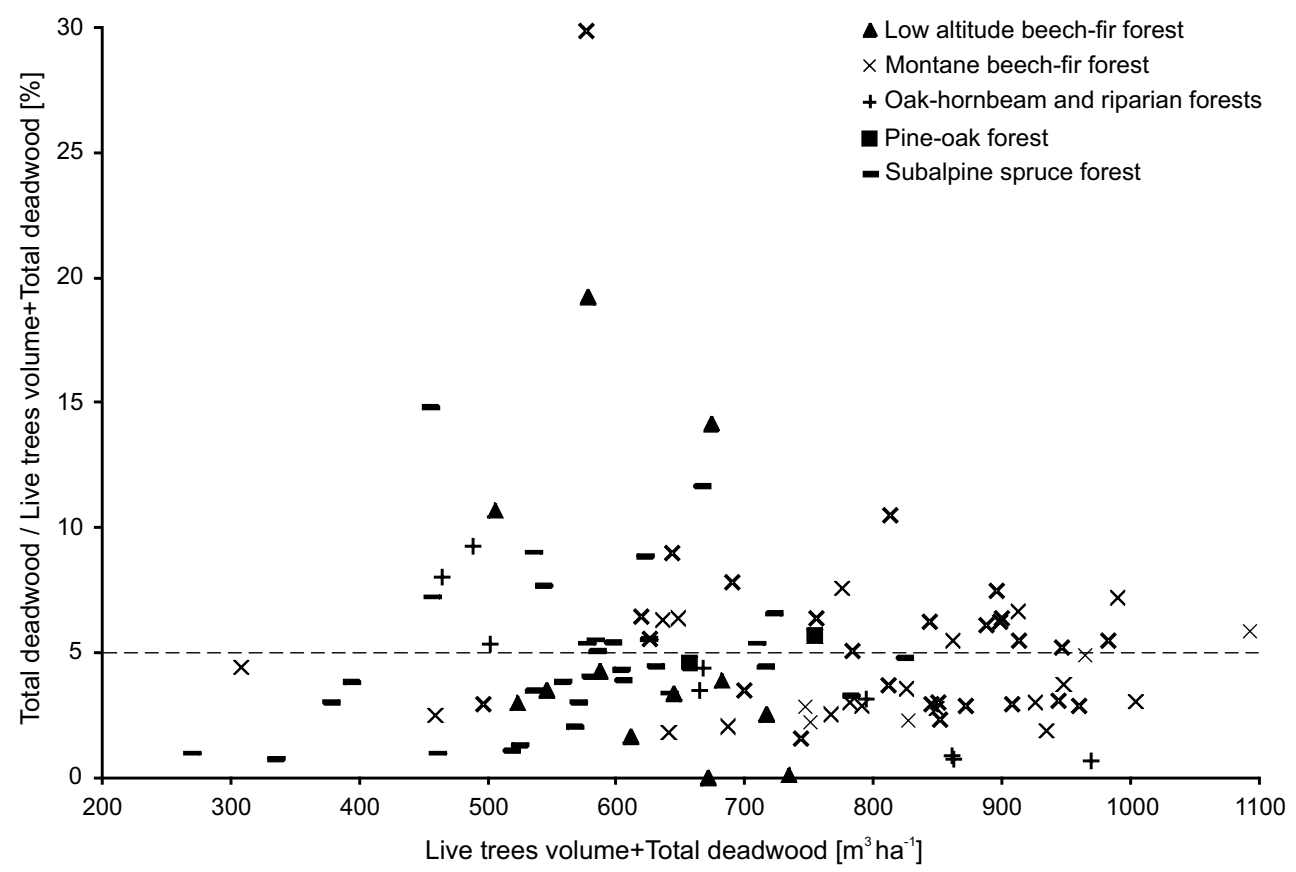

Fig. 3 Relationship between deadwood volume and combined volume of deadwood and live trees for the various studies (the dashed horizontal line indicates the mean total deadwood/combined volume of deadwood and live trees ratio (\%), which is 5.0\%)

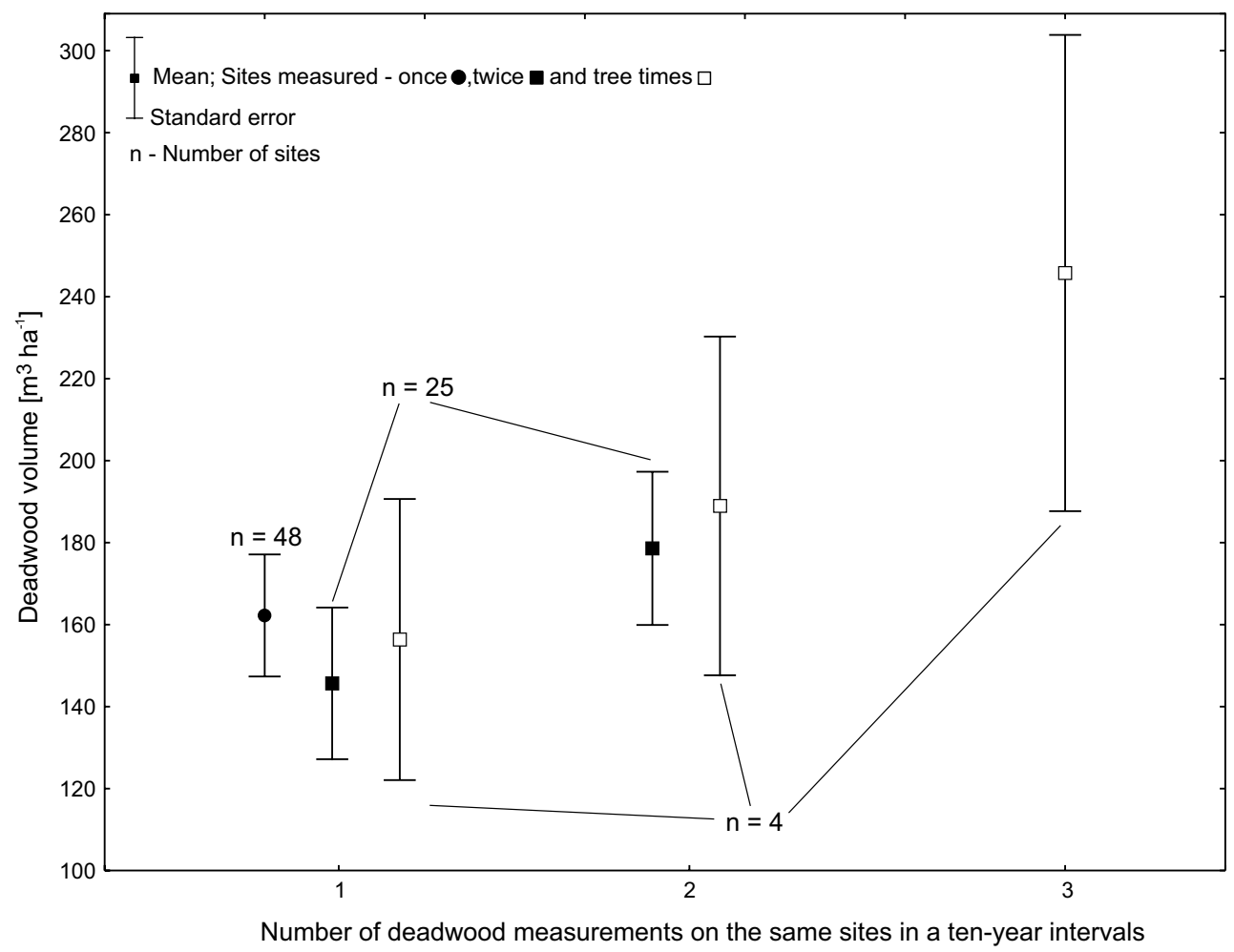

Fig. 4 Relationship between the number of measurements on the same sites and deadwood volume

$65.3 \mathrm{~m}^{3} / \mathrm{ha}\left(\mathrm{SD}=49.2 \mathrm{~m}^{3} / \mathrm{ha}\right)$. In different studies, standing deadwood accounted for $0.4-100.0 \%$ of total deadwood volume, with the mean being $40.1 \%(\mathrm{SD}=17.9 \%)$. Significant differences in standing deadwood volume were found between different forest types (Kruskal-Wallis; $H=30.8$; $p<0.001$ ) (Fig. 8). No differences were identified between 


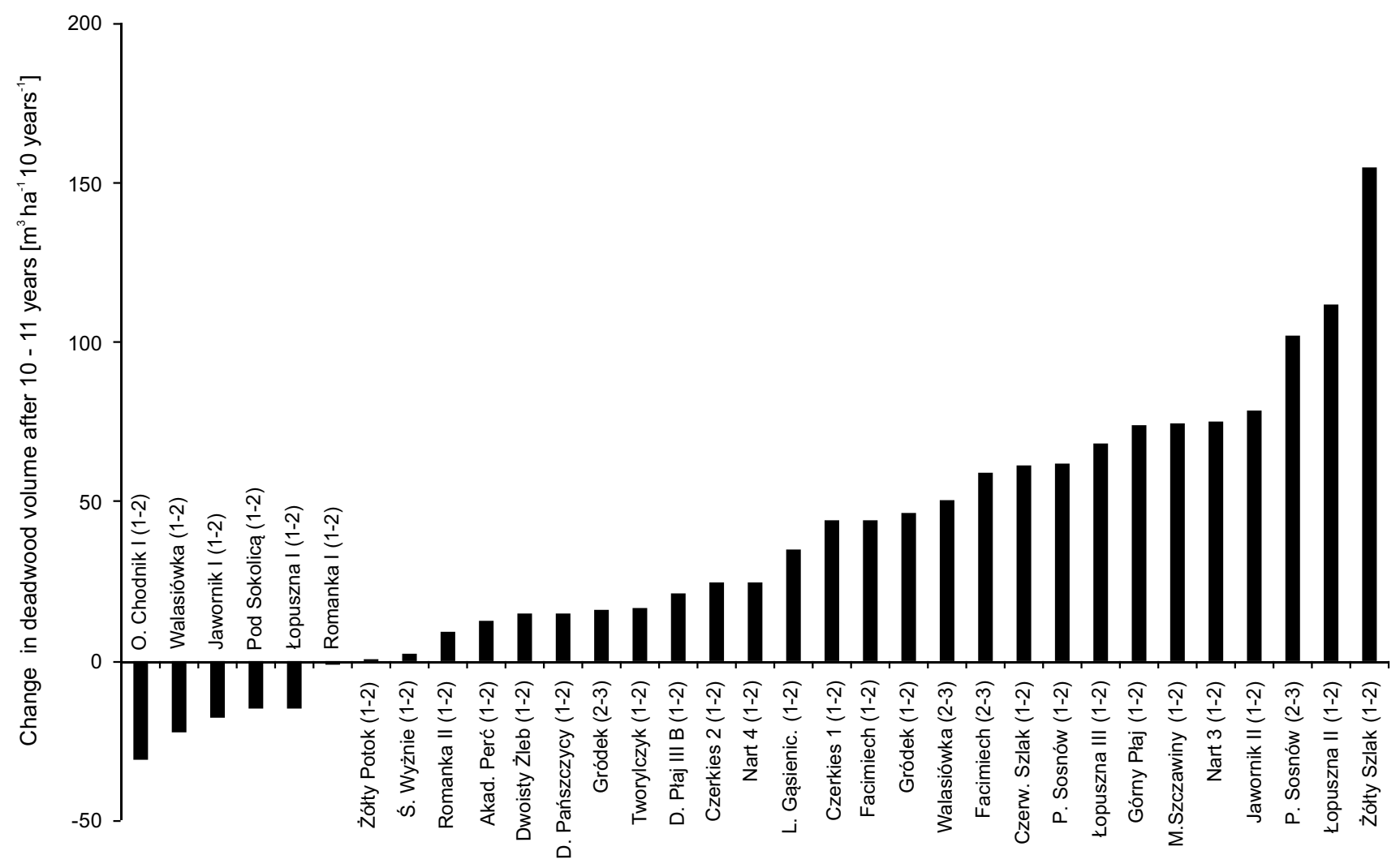

Names of sites according to Appendix (Number of deadwood measurements on the same sites)

Fig. 5 Changes in deadwood volume between consecutive measurements on the same sites at 10-11 year intervals

low-altitude beech-fir forests and both high-altitude forest types. In the remaining cases, the differences were significant, with the highest average standing deadwood proportion being $50.1 \%$ in subalpine spruce forests and the lowest proportion being $16.0 \%$ in oak-hornbeam and riparian forests.

Among the studied models, the lowest RMSE (73.7) was exhibited by the one consisting of elevation a.s.l. and input of dead trees (Table 1). The linear regression model with the best fit showed that these two variables are independent predictors of the dependent variable $(\mathrm{p}<0.05)$. An increase in the variable "elevation a.s.l." by $x$ units, when all other predictive variables were kept constant, translated into a mean increment in the dependent variable by $1.037 x-0.001 x^{2}$ units. The variable "input of dead trees," ranging from 2.0 to $148.4 \mathrm{~m}^{3} / \mathrm{ha} / \mathrm{decade}$ was also significant. In this case, an increase by 1 unit resulted in a mean increase in the dependent variable by 1.651 units, when all else being constant. However, the dependent variable was affected to the greatest degree by "elevation a.s.l," as indicated by the largest absolute values of beta coefficients. The $\mathrm{R}^{2}$ coefficient for this model was $63.54 \%$. Thus, the remaining $36.44 \%$ is attributable to variables that were not included in the model, as well as to random effects.
Models developed for all elevations a.s.l. (all forest types), but excluding the input of dead trees (due to a lack of data for low altitude beech-fir forest and oak-hornbeam and riparian forests) led to higher RMSE values (the lowest RMSE was 83.7, with the model consisting of the following variables: volume of living trees, duration of conservation, stage of forest development, and forest type)

\section{Discussion}

\section{Deadwood volume and forest type}

Numerous studies have addressed deadwood volume in Poland; however, they mostly focused on the southern and eastern regions of the country, where most natural forests and national parks with high forest cover are located. Relatively few papers have been devoted to Pinus sylvestris stands, which constitute the prevalent forest communities in Poland, but predominantly of managed character. The most substantial body of data is available for the Carpathian Mountains, and so tree stands from that area exert the greatest effect on the mean values obtained in this review. An 


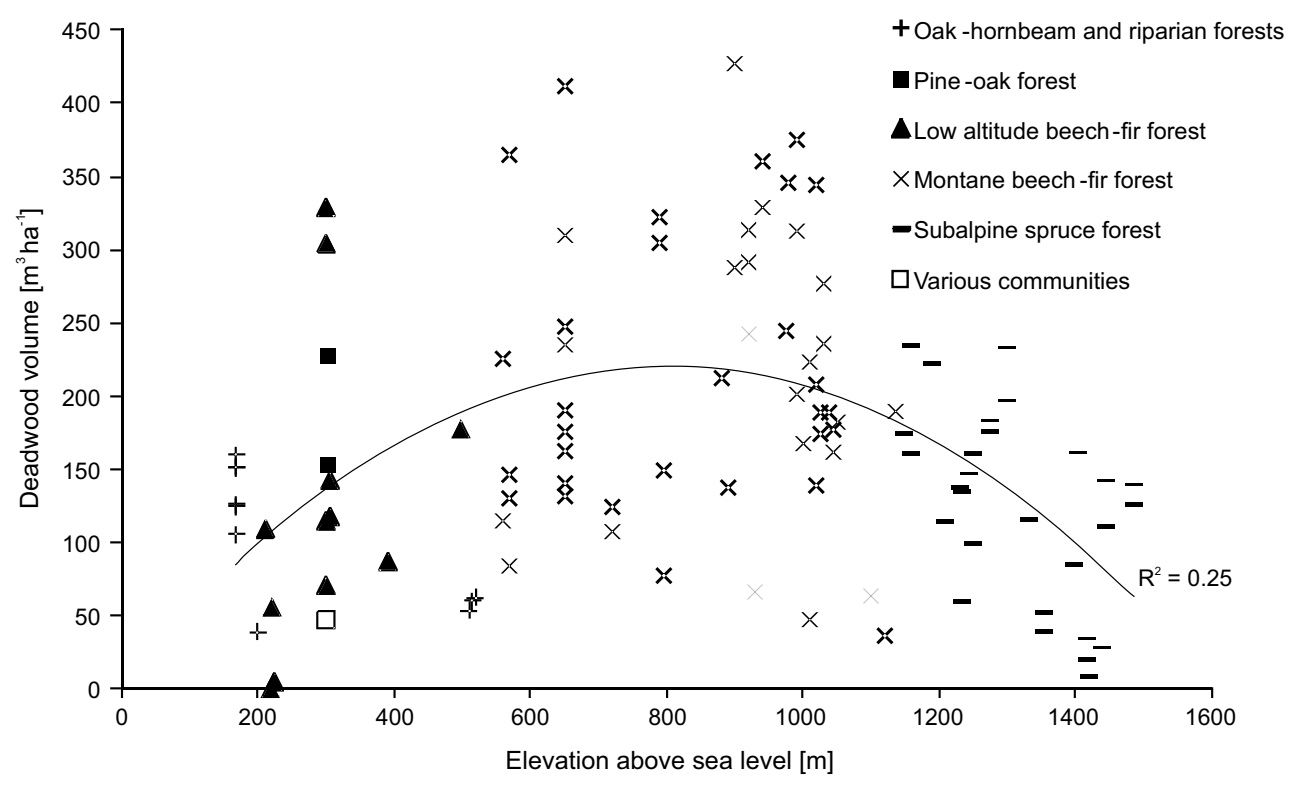

Fig. 6 Relationship between sites elevation a.s.l. and deadwood volume

Fig. 7 Deadwood volume in different forest types (values with different letters differ significantly at $p<0.05$ as evaluated by the post hoc Scheffe test, only the latest measurements on sites were analyzed, $n=75$ )

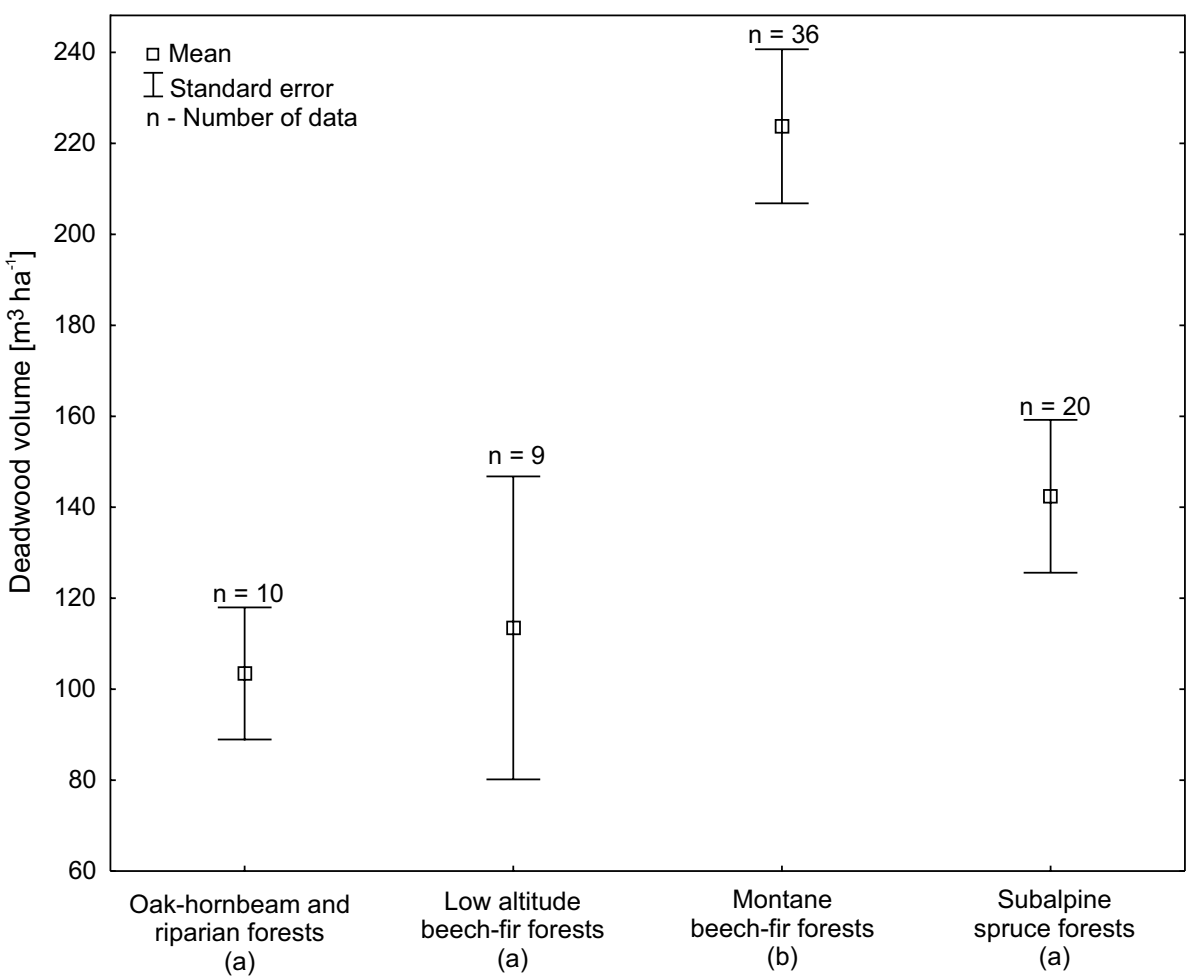

Forest type (b) (a) important feature of the analyzed data is that they are derived from studies applying diverse methodologies, mostly differing in the number and size of sampling plots. The majority of studies used a single sampling plot, usually ranging from 0.25 to 1 ha, while a number of other studies used grids of several dozen to hundreds of sampling plots with a size of several hundreds $\mathrm{m}^{2}$ each. Finally, in a few studies measurements were conducted along transects of varying lengths. The other aspect is the location of sites. In some studies, they were distributed randomly, but in most cases their location was not random. Nevertheless, researchers generally selected sites in fragments of forests of primeval character, 
Fig. 8 Share of standing deadwood in total deadwood volume by forest type (values with different letters differ significantly at $p<0.05$ as evaluated by the nonparametric Kruskal-Wallis test and corrected with the post hoc test for number of comparisons)

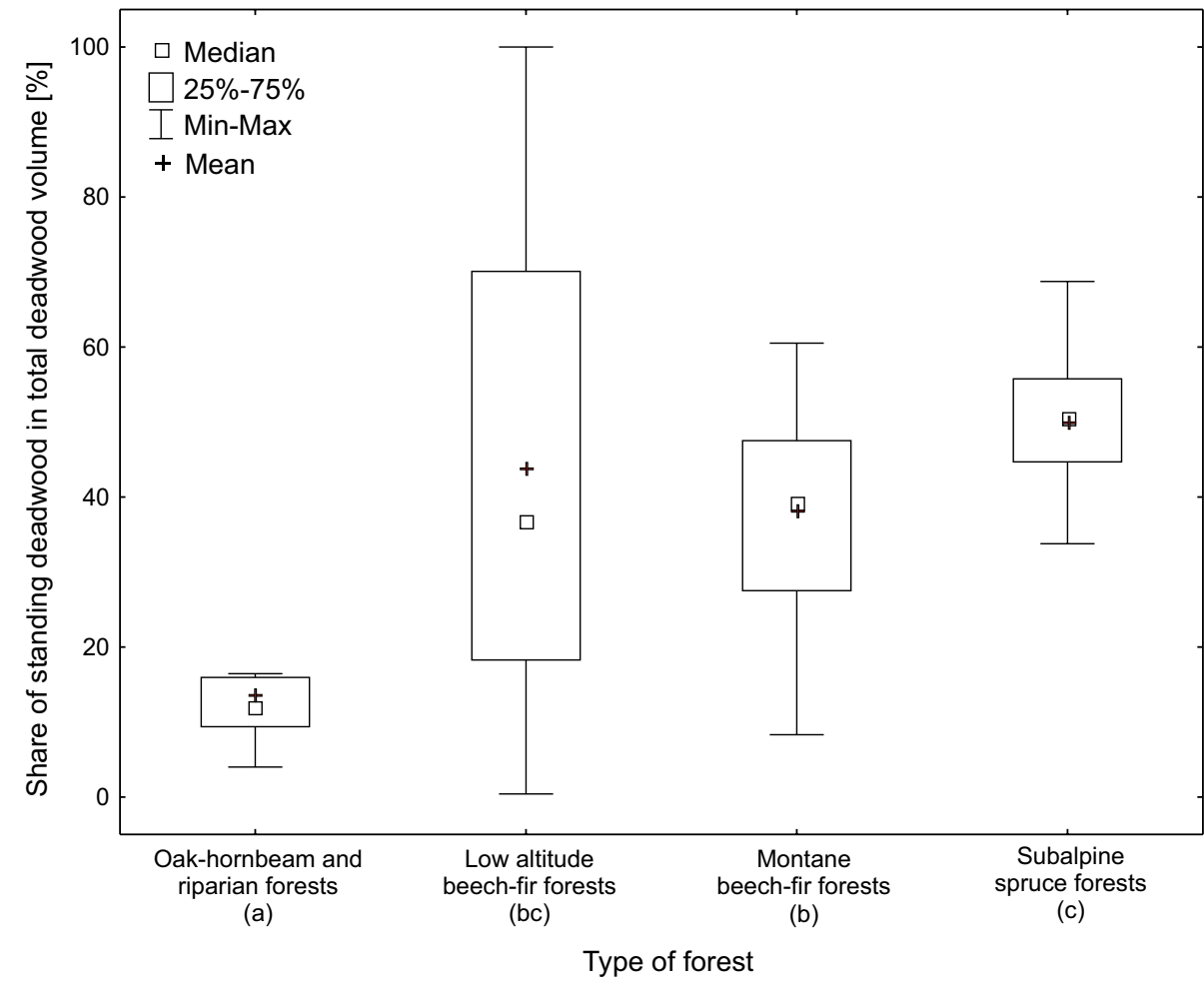

Table 1 Linear regression results for two forest types: montane beech-fir forest and subalpine spruce forest

\begin{tabular}{|c|c|c|c|c|c|}
\hline Variable & Beta coefficient & Regression parameter & $95 \% \mathrm{CI}$ & & $p$ \\
\hline Constant & - & -529.609 & -914.249 & -144.969 & 0.009 \\
\hline Elevation a.s.1 (m) & 3.192 & 1.037 & 0.438 & 1.635 & 0.001 \\
\hline Elevation a.s. $1^{2}(\mathrm{~m})$ & -3.114 & -0.001 & -0.001 & 0 & 0.002 \\
\hline Volume of living trees $\left(\mathrm{m}^{3} / \mathrm{ha}\right)$ & -0.161 & 0.116 & -0.119 & 0.351 & 0.319 \\
\hline Duration of conservation (year) & 0.275 & 1.472 & -0.003 & 2.946 & 0.05 \\
\hline Input of dead trees $\left(\mathrm{m}^{3} / \mathrm{ha} /\right.$ decade $)$ & 0.689 & 1.651 & 0.7 & 2.603 & 0.001 \\
\hline \multicolumn{6}{|l|}{ Stage of forest development } \\
\hline Growing up & - & Reference & & & \\
\hline Optimum & -0.118 & -22.736 & -79.178 & 33.706 & 0.415 \\
\hline Breakup & -0.341 & -69.361 & -148.956 & 10.234 & 0.085 \\
\hline
\end{tabular}

Normal distribution of residuals and homoscedasticity of variances were satisfied ( $p>0.05$ Shapiro-Wilk's and Breusch-Pagan's tests)

making sure they varied in terms of the stage of development. In most studies deadwood was not the main focus of research, but rather one of many variables measured. In the future, it would be advisable to increase the studied areas and promote a more uniform methodology.

Deadwood volume in unmanaged forests often depends on forest type, which is in turn determined by climatic and site conditions, as they influence the distribution of plant communities (Lombardi et al. 2012; Karjalainen and Kuuluvainen 2002). However, this has not been corroborated by some studies. Larrieu et al. (2014) reported that deadwood availability remained more or less stable throughout the silvigenetic cycle, both in terms of quantity and diversity, and whether the forests were dominated by broadleaves or conifers. However, numerous studies from different geographic regions of the world showed large variation in the amount of deadwood depending on forest type and stage of forest development (Burrascano et al. 2008; Lombardi et al. 2008, 2012; Karjalainen and Kuuluvainen 2002; Mataji et al. 2014). For example, in natural spruce-beech forest stands in the Krkonoše National Park, Czech Republic deadwood measurements revealed from 27.7 to $241.6 \mathrm{~m}^{3} /$ ha depending on the site and year of survey. Higher deadwood volumes were reported from silver fir-beech forests in the Dinaric 
Mountains, Slovenia, where the highest and lowest concentrations of CWD for virgin forests were found in the regeneration phase $\left(626.0 \mathrm{~m}^{3} / \mathrm{ha}\right)$ and the juvenile phase $\left(248.3 \mathrm{~m}^{3} /\right.$ ha), respectively (Debeljak 2006). The results obtained for different Carpathian regions generally indicated high deadwood volumes, which was also corroborated by Polish data. A primeval beech forest in the Ukrainian Carpathians had a deadwood volume of $162.5 \mathrm{~m}^{3} /$ ha (Hobi et al. 2015), while in a silver fir-beech virgin forest in the Southern Carpathians of Romania that value amounted to $134.9 \mathrm{~m}^{3} / \mathrm{ha}$ (Petritan et al. 2015).

A review of deadwood volume in reserves representing almost the entire European range of beech was published by Christensen et al. (2005). On the basis of the data from 86 sites, they reported a mean deadwood volume of $130 \mathrm{~m}^{3} /$ ha, varying from almost nothing $\left(6 \mathrm{~m}^{3} / \mathrm{ha}\right)$ to $550 \mathrm{~m}^{3} / \mathrm{ha}$. Among lowland and montane forests with different duration of conservation, the highest amount of deadwood $\left(220 \mathrm{~m}^{3} /\right.$ ha) was found in mountain nature reserves that had been protected for more than 50 years, while the corresponding value for those protected for a shorter time was only $117 \mathrm{~m}^{3} /$ ha. The difference was much smaller for lowland nature reserves (132 vs. $99 \mathrm{~m}^{3} / \mathrm{ha}$, respectively). The Polish results are consistent with the above data, although in this case the duration of conservation was not found to have a direct effect on deadwood volume. In the present review, montane beech-fir forests were much richer in deadwood than low altitude beech-fir stands. It should also be noted that data from Poland are closer to the aforementioned results for forests with a 50-year conservation period. The differences between montane and low altitude beech-fir forests may be attributable to greater site productivity (live tree volume + total deadwood volume) in montane stands, as well as to the fact that they often contain a minor component of spruce, which may remain standing for many years after its death, slowly decomposing and adding to deadwood at a given site (Zielonka 2006b). Also dead trees already present before a conservation regime was introduced may contribute to the differences. In mountainous areas, the volume of remaining dead trees may be greater due to difficult terrain hindering their removal (Bujoczek et al. 2015).

The Polish results from montane forests are also similar to data from spruce stands in other countries. A deadwood inventory conducted in the montane zone in central Slovakia reported a mean volume of $143.5 \mathrm{~m}^{3} / \mathrm{ha}$ (Holeksa et al. 2007). Data from the Czech Šumava National Park from the year 2002 indicated 311 and $156 \mathrm{~m}^{3} / \mathrm{ha}$ (Svoboda and Pouska 2008), while a survey of a Japanese old-growth subalpine coniferous forest revealed $217.9 \mathrm{~m}^{3} / \mathrm{ha}$ (Fukasawa et al. 2014). In boreal natural forests in the Petersburg region, deadwood volume ranged from 32 to $326 \mathrm{~m}^{3} / \mathrm{ha}$ (Shorohova and Shorohov 2001). Much lower values were recorded in the Italian Alps $\left(23 \mathrm{~m}^{3} / \mathrm{ha}\right)$ in a reserve that had been established only 14 years prior to measurement (Motta et al. 2006).

In addition to the studies included in this work, in Poland there are other sites with considerable quantities of deadwood, but they are not always strictly protected (e.g., landscape parks with a lower protection status). Survey results indicating high deadwood volumes (33-166 $\mathrm{m}^{3} / \mathrm{ha}$ ) show that deadwood has not been removed from some of those areas for decades (Karczmarski and Bąk 2010; Maślak and Orczewska 2010). Some accumulations of deadwood $\left(27 \mathrm{~m}^{3} /\right.$ ha) are also found in certain areas of managed forests, far exceeding the mean value for Polish forests of $5.8 \mathrm{~m}^{3} / \mathrm{ha}$ (WISL 2014; Bujoczek and Bujoczek 2016). Also protection zones with a radius of several hundred meters established temporarily around endangered species pursuant to the Polish law exhibit above-average deadwood volumes. In such zones, forest management treatments, including deadwood removal, are prohibited (Banaś et al. 2014).

The forests included in this study differ in terms of their development stages (Korpel 1989, 1995), which are linked to the proportion and size of trees dying in a given period. That in turn translates into the input of dead trees, a variable exerting a significant effect on the volume of deadwood identified on the studied sites. However, the input of dead trees was reported only for two forest types with the observation period typically amounting to approximately 10 years. Thus, regression models may be improved if information about tree mortality over a longer period of time is included. It would also be useful to take into account the species composition of the dying trees as this factor seems likely to affect the decay rates on the studied sites in view of the increasing body of knowledge on this subject (Kahl et al. 2017), but currently such data are not available.

\section{Duration of conservation and the share of standing deadwood}

The average deadwood volume for all the sites was $172.0 \mathrm{~m}^{3} /$ ha, ranging from 0 to $427 \mathrm{~m}^{3} /$ ha (with the highest results observed for a forest in the breakup stage of development). While the higher spectrum of results is uncontroversial, the lowest values are surprising as according to the cited authors deadwood had not been removed from the studied sites for at least 21 years (Karczmarski and Kunz 2010). The absence of deadwood could be partially explained by the small size of sample plots, whose distribution was often not random, or by the fact that some tree stands could have been in the optimum stage of development, in which few trees die (Korpel 1989, 1995). A similarly broad range of deadwood volume, including very low values for some sites, was reported in the review paper by Christensen et al. (2005). In that work, Christensen et al. found a significant correlation with the age of the reserve. Other reports also indicated that the duration 
of conservation significantly affects deadwood accumulation (Vandekerkhove et al. 2009; Bouget et al. 2014; Paillet et al. 2015). Although such a relationship was also expected to emerge in the present work, it was not confirmed directly, which is attributable to several factors. First, it is often very difficult to accurately determine the duration of strict protection. On some sites, it may not have been continuous, being interrupted by periods of sanitary thinning following insect outbreaks or windthrows (such data are often absent from publications). On other sites, forest management treatments may have been discontinued long before the initiation of strict protection (Some sites did not reveal signs of human intervention.) Furthermore, there are areas which were incorporated into national parks years after the latter had been established, while other conservation areas merged, which makes it impossible to precisely determine for how long they had been set aside. Second, the expected correlation between deadwood volume and conservation duration may have been disturbed by biotic and abiotic factors dramatically increasing the amount of deadwood soon after the introduction of strict protection.

However, a relationship between deadwood volume and the duration of conservation was identified on sites subjected to repeated measurements. An increase was found for more than $3 / 4$ of the sites, with a maximum of $155 \mathrm{~m}^{3} / \mathrm{ha} /$ decade. The observed significant increments in deadwood volume were usually associated with a decline in stand volume. A decrease in the amount of deadwood is typically attributable to decay outpacing mortality. It should be noted that the decay rate is largely species-specific; for instance, beech decompose faster than spruce (Holeksa et al. 2008; Rock et al. 2008; Müller-Using and Bartsch 2009; Herrmann et al. 2015). Other contributing factors include local climatic conditions and the presence of organisms decomposing organic matter (Zhou et al. 2007). Furthermore, the wood decay rate may vary between the four analyzed forest types due to their different thermal and moisture conditions. In the present review, the maximum decrease in deadwood volume was $31 \mathrm{~m}^{3} / \mathrm{ha} /$ decade. Similar decay-related changes in deadwood volume were described for the Krkonoše National Park by Vacek et al. (2015), who conducted measurements on a permanent research plot over a 40 -year period at 5-year intervals. If any decreases in the amount of CWD were observed, they were relatively small for both dominant species, that is, spruce and beech. On the other hand, the dynamics of standing deadwood volume revealed a shorttermed rise followed by a drop back to previous levels.

Deadwood volume and diversity are often used as biodiversity indicators (Gao et al. 2015). From the point of view of organisms living in and feeding on deadwood, of great importance is the position of dead trees. Thus, one of the main parameters of deadwood is the relative proportion of its standing and downed forms, which is largely determined by the species composition of tree stands and the causes of tree mortality. High percentages of snags are reported from areas affected by insect outbreaks or fires (Pedlar et al. 2002; Bujoczek et al. 2015; Franklin et al. 2015). Insect outbreaks, which often cause a die-off of spruces, increase the quantity of snags, leading to a higher proportion of standing deadwood in subalpine spruce forests as compared to other types of tree stands (Bobiec 2002; Bujoczek et al. 2015). Indeed, dead spruces may remain standing for dozens of years. Holeksa et al. $(2006,2008)$ reported that $40 \%$ of such snags may still be found in the third decade after dying. In the present study, the largest mean volume of standing deadwood was found for tree stands with a higher presence of spruce. On the other hand, spruce may also contribute to rapid changes in the standing to downed deadwood ratio due to the high susceptibility of this species to strong winds. For instance, a study from the lowland tree stands of the Białowieża Forest indicated that approximately 50\% of spruces were dead at the time of falling, in contrast to almost all deciduous trees (Faliński 1978).

\section{Conclusions}

The results of studies included in the present review indicate very high variation in the volume of deadwood in strictly protected forests and forests of primeval character in Poland. This is mostly attributable to differences in the dynamics of tree mortality between sites in the years preceding deadwood measurements. It should also be noted that $63.54 \%$ of the variation of the dependent variable was explained by the factors included in the model, with the remaining variation being linked to other factors, random effects, or methodological discrepancies between studies. Due to this, future research should include more variables concerning the studied sites. In addition, it would be beneficial to standardize deadwood measurement methods.

Acknowledgements This Research was financed by the Ministry of Science and Higher Education of the Republic of Poland. The authors thank the anonymous Reviewers for their comments, which have greatly contributed to the improvement of an earlier version of this manuscript.

Open Access This article is distributed under the terms of the Creative Commons Attribution 4.0 International License (http://creativeco mmons.org/licenses/by/4.0/), which permits unrestricted use, distribution, and reproduction in any medium, provided you give appropriate credit to the original author(s) and the source, provide a link to the Creative Commons license, and indicate if changes were made.

\section{Appendix}

See Table 2. 


\begin{tabular}{|c|c|c|c|c|c|c|c|c|c|c|c|c|c|c|c|c|c|c|c|c|c|c|c|c|}
\hline รәวนวдәృу & 9 & 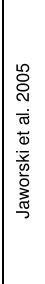 & 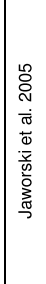 & 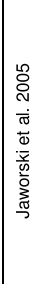 & 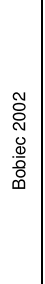 & 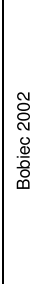 & 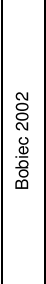 & 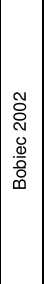 & 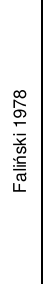 & 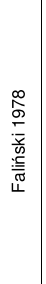 & 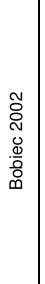 & 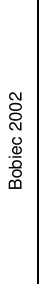 & 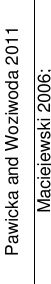 & 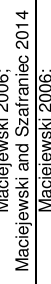 & 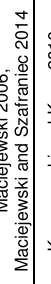 & 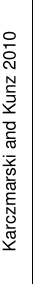 & 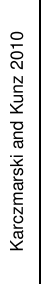 & 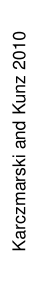 & 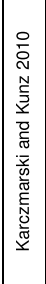 & \begin{tabular}{|l} 
\\
\\
$N$ \\
$\tilde{D}$ \\
$\stackrel{N}{\tilde{N}}$ \\
$\frac{\tilde{N}}{\infty}$ \\
\end{tabular} & 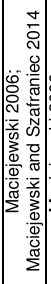 & 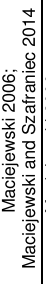 & 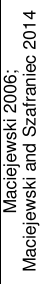 & 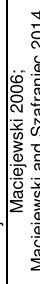 \\
\hline 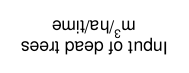 & 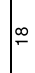 & & & & & & & & & & & & & & & & & & & 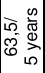 & & & & \\
\hline 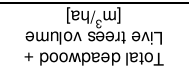 & $\neq$ & $\frac{\infty}{\infty}$ & ๙ิ & $\frac{9}{\infty}$ & \begin{tabular}{|l|l}
$\substack{\infty \\
\infty \\
\sim}$ &
\end{tabular} & $\begin{array}{l} \\
0 \\
\end{array}$ & i. & $\hat{\ddot{\theta}}$ & & & 吉 & 总 & & G & 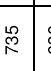 & : & है & 今ి & 总 & 岕 & $\frac{0}{10}$ & ฐิ & 䓌 & \&్ \\
\hline 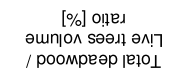 & $\because$ & 总 & 兽 & 总 & 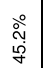 & 空 & 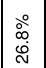 & 今. & & & $\stackrel{\circ}{\stackrel{\circ}{\rho}}$ & ڤั̊ & & @ं & $\frac{\circ}{\dot{g}}$ & 兽 & :̊․ㅇ & $\begin{array}{l}\grave{2} \\
\vdots \\
0\end{array}$ & $\frac{\circ}{\stackrel{\circ}{\circ}}$ & \begin{tabular}{|l|} 
\\
$\dot{\circ}$ \\
$\stackrel{\circ}{\sigma}$
\end{tabular} & 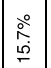 & \begin{tabular}{|l|}
$\circ$ \\
$\stackrel{\circ}{N}$ \\
$\stackrel{\sim}{N}$
\end{tabular} & $\stackrel{\stackrel{\circ}{\circ}}{\stackrel{\rho}{\risingdotseq}}$ & ڤั̊ \\
\hline 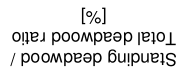 & 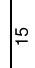 & $\begin{array}{l}\circ \\
o \dot{m} \\
0\end{array}$ & 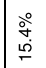 & $\begin{array}{l}\stackrel{\circ}{\circ} \\
\stackrel{\circ}{\circ}\end{array}$ & $\begin{array}{l}\stackrel{\circ}{\stackrel{\circ}{0}} \\
\stackrel{\leftrightarrow}{\circ}\end{array}$ & $\begin{array}{l}\stackrel{\circ}{0} \\
\dot{+}\end{array}$ & $\begin{array}{l}\stackrel{\circ}{\hat{R}} \\
\dot{r}\end{array}$ & \begin{tabular}{|}
$\stackrel{\circ}{\circ}$ \\
$\stackrel{+}{=}$
\end{tabular} & & & $\stackrel{\circ}{\stackrel{\circ}{\risingdotseq}}$ & ণे & 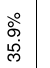 & & 商 & 宫 & & 兽 & 孛 & 总 & & \begin{tabular}{|l|}
$\circ$ \\
$\vdots$ \\
\end{tabular} & & $\stackrel{\circ}{\stackrel{9}{\oplus}}$ \\
\hline 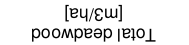 & \pm & in & is & in & $\stackrel{\sim}{\sim}$ & $\stackrel{\stackrel{n}{N}}{\sim}$ & $\stackrel{\circ}{\circ}$ & in & & & $\stackrel{\mathscr{N}}{\sim}$ & $\stackrel{\circ}{\circ}$ & g్ & $\stackrel{g}{+}$ & $\overline{\mathrm{N}}$ & $\stackrel{\overbrace{}}{\text { g }}$ & ○ & in & i & $\infty$ & 8 & $\stackrel{\sim}{\rightleftharpoons}$ & స్ & స్ల \\
\hline $\begin{array}{c}{[\varepsilon ч / \varepsilon ш]} \\
\text { роомреәр рәимоб }\end{array}$ & $m$ & 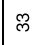 & in & $\stackrel{\infty}{\sim}$ & $\stackrel{\widehat{N}}{\sim}$ & $\stackrel{\text { I }}{\sim}$ & $\overline{0}$ & $\stackrel{\mathscr{m}}{\sim}$ & 8 & $\pi$ & $\stackrel{\cong}{=}$ & $\stackrel{\text { 导 }}{\stackrel{5}{+}}$ & $\stackrel{\mathscr{N}}{N}$ & & $\stackrel{n}{=}$ & $\stackrel{8}{\circ}$ & $\circ$ & 0 & 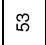 & : & & $\mathscr{q}$ & & $\stackrel{\mathscr{\infty}}{\sim}$ \\
\hline $\begin{array}{c}{[\mathrm{[ey/z} w]} \\
\text { роомреәр бuіpuets }\end{array}$ & $\simeq$ & $\stackrel{\infty}{\sim}$ & 0 & 0 & $\stackrel{\sim}{\sim}$ & ம & in & $\stackrel{\infty}{\sim}$ & & & \pm & $\stackrel{\curvearrowleft}{\leftarrow}$ & $\stackrel{+}{\leftarrow}$ & & 응 & ब्ल & $\circ$ & in & 0 & i & & 8 & & ? \\
\hline 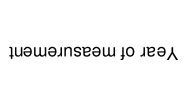 & $\mp$ & : & : & : & 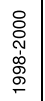 & 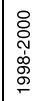 & 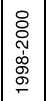 & 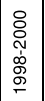 & $\begin{array}{l}\stackrel{ \pm}{\leftrightarrows} \\
\stackrel{\circ}{\circ}\end{array}$ & 去 & 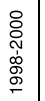 & 离 & ํㅜㄴ & 总 & 敵 & 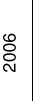 & : & : & : & 啇 & $\stackrel{\text { g }}{\stackrel{g}{g}}$ & 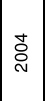 & $\stackrel{\text { 萼 }}{\stackrel{2}{2}}$ & 兽 \\
\hline 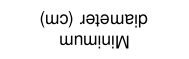 & $\div$ & $\infty$ & $\infty$ & $\infty$ & $\sigma$ & + & $\sigma$ & $\theta$ & & & ナ & ナ & in & n & $\wedge$ & $\infty$ & $\infty$ & $\infty$ & $\infty$ & $\wedge$ & $r$ & $\wedge$ & $r$ & $\wedge$ \\
\hline 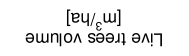 & o & $\begin{array}{l}\infty \\
\stackrel{\infty}{R}\end{array}$ & \begin{tabular}{|l|}
$\bar{\Xi}$ \\
$\infty$
\end{tabular} & छ & $\stackrel{\infty}{\infty}$ & 兽 & 总 & $\frac{0}{10}$ & & & 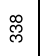 & 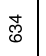 & & ชั & in & î & : & $\widetilde{\Xi}$ & $\widehat{\widehat{N}}$ & f & $\bar{q}$ & in & 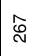 & $\stackrel{\infty}{m}$ \\
\hline 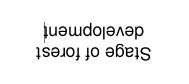 & $\infty$ & $\begin{array}{l}0 \\
0 \\
0 \\
0 \\
0\end{array}$ & ஜ & O⿱ & 㤎: & 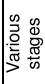 & 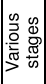 & 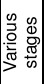 & & 5 & 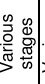 & 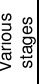 & & & & $\stackrel{n}{m}$ & \&̊ & 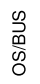 & 垈 & o & & & & \\
\hline 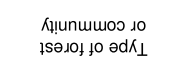 & n & 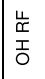 & 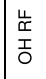 & \begin{tabular}{|l} 
崖 \\
I \\
\end{tabular} & 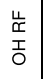 & 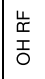 & 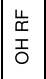 & 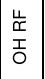 & 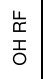 & $\begin{array}{l}\stackrel{u}{\underline{x}} \\
\text { I } \\
\text { I }\end{array}$ & 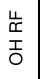 & 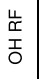 & $\begin{array}{l}\frac{u}{\underline{x}} \\
\text { İ }\end{array}$ & 잉 & 혐 & 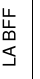 & 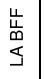 & 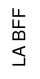 & 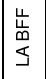 & 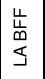 & 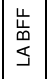 & 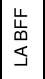 & 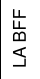 & 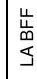 \\
\hline '|se uo!̣enㅋㅋ & 0 & $\frac{0}{10}$ & ్ㅗ & $\frac{20}{i n}$ & $\mid$ & 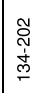 & 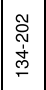 & 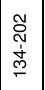 & 잉 & $\stackrel{1}{9}$ & 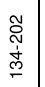 & 产 & ڤั & 娄 & 管 & 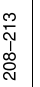 & $\begin{array}{l}\bar{N} \\
\stackrel{N}{N}\end{array}$ & $\begin{array}{l}\stackrel{N}{N} \\
\text { N్ } \\
\text { N. }\end{array}$ & $\begin{array}{l}\bar{N} \\
\stackrel{\omega}{\infty} \\
\stackrel{N}{N}\end{array}$ & \begin{tabular}{|l|}
0 \\
0 \\
$\vdots$ \\
$o$ \\
$o$ \\
0 \\
\end{tabular} & 号 & 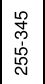 & 点 & $\begin{array}{l}\text { 号 } \\
\text { 㟧 } \\
\text { N }\end{array}$ \\
\hline 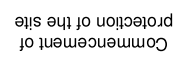 & is & $\frac{9}{2}$ & $\frac{9}{2}$ & $\frac{9}{2}$ & $\overline{\bar{\alpha}}$ & 离 & $\overline{\bar{\alpha}}$ & $\overline{\bar{\alpha}}$ & $\overline{\bar{\alpha}}$ & $\overline{\bar{\alpha}}$ & $\overline{\bar{\alpha}}$ & $\overline{\bar{N}}$ & 足 & 쑝 & 용 & $\stackrel{\stackrel{\rho}{\infty}}{\stackrel{\infty}{\circ}}$ & $\stackrel{\stackrel{\infty}{\infty}}{\sim}$ & \begin{tabular}{|l}
$\stackrel{\infty}{\infty}$ \\
$\stackrel{\infty}{\infty}$ \\
$\stackrel{2}{*}$
\end{tabular} & 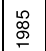 & 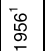 & 空 & 岕 & $\stackrel{\leftrightarrow}{\stackrel{\leftrightarrow}{\circ}}$ & s. \\
\hline $\begin{array}{l}\text { poyrew } \\
\text { 6uldures }\end{array}$ & t & 8 & 8 & 0 & $\stackrel{\rho}{J}$ & $\mathscr{S}$ & $\mathscr{\infty}$ & $\stackrel{\infty}{J}$ & 8 & 8 & $\stackrel{D}{I}$ & $\stackrel{\mathscr{S}}{I}$ & $\stackrel{\infty}{J}$ & y & \& & y & 8 & $\ddot{0}$ & 8 & is & $\%$ & 8 & 8 & \% \\
\hline 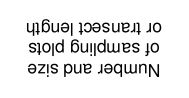 & m & 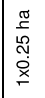 & 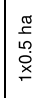 & 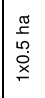 & $\begin{array}{l}E \\
\vdots \\
\stackrel{0}{-}\end{array}$ & $\begin{array}{l}E \\
\circ \\
\circ\end{array}$ & 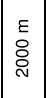 & \begin{tabular}{ll}
$E$ \\
\\
$\vdots$ \\
\hdashline
\end{tabular} & \begin{tabular}{|l}
$\frac{\pi}{x}$ \\
$\frac{x}{x}$
\end{tabular} & $\begin{array}{l}\frac{\pi}{2} \\
\underline{x} \\
\underline{x}\end{array}$ & $\begin{array}{l}\varepsilon \\
\stackrel{0}{\circ} \\
\square\end{array}$ & $\begin{array}{l}E \\
\stackrel{2}{\circ} \\
\stackrel{-}{-}\end{array}$ & 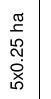 & 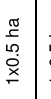 & 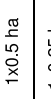 & 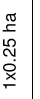 & 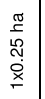 & 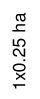 & 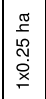 & 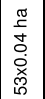 & 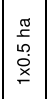 & 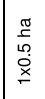 & 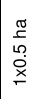 & 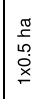 \\
\hline 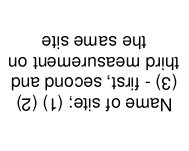 & N & 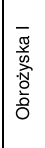 & 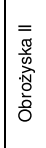 & 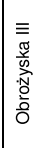 & 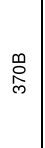 & $\begin{array}{l}0 \\
0 \\
0 \\
m\end{array}$ & 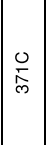 & $\frac{R}{p}$ & 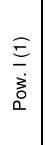 & $\begin{array}{l}\widehat{\widehat{a}} \\
\frac{\bar{z}}{3} \\
0\end{array}$ & $\frac{\bar{\alpha}}{\alpha}$ & $\frac{N}{\alpha}$ & 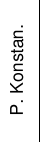 & $\begin{array}{l}\text { E } \\
\text { m } \\
\text { 䎡 }\end{array}$ & 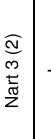 & - & $=$ & $\equiv$ & $\geq$ & - & 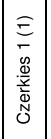 & 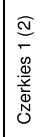 & 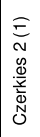 & 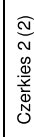 \\
\hline 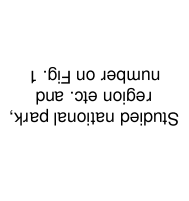 & - & 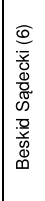 & 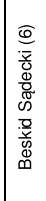 & 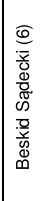 & 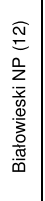 & 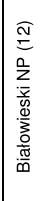 & 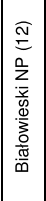 & 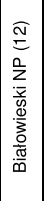 & 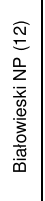 & 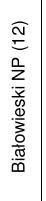 & 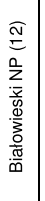 & 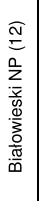 & $\underset{0}{0}$ & 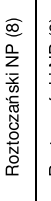 & 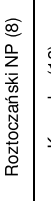 & 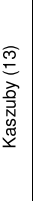 & 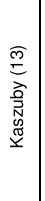 & 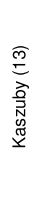 & 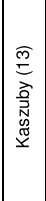 & 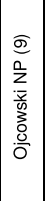 & 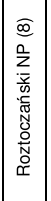 & 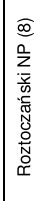 & 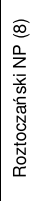 & 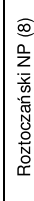 \\
\hline
\end{tabular}




\begin{tabular}{|c|c|c|c|c|c|c|c|c|c|c|c|c|c|c|c|c|c|c|c|c|c|c|c|c|c|c|c|c|}
\hline 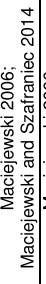 & 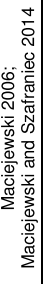 & 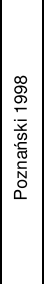 & & 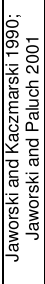 & 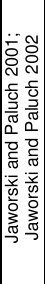 & 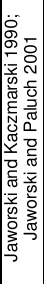 & 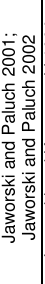 & & 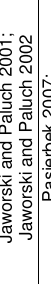 & & 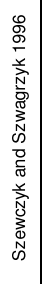 & 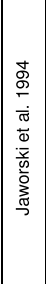 & 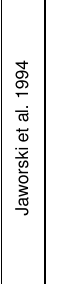 & 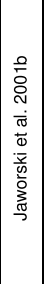 & 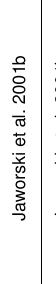 & 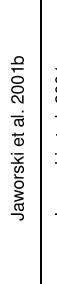 & 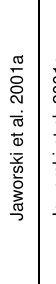 & 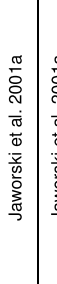 & & & & & & 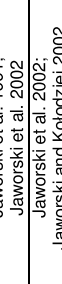 & & 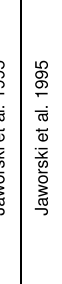 & 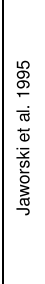 & 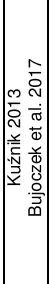 \\
\hline & & & & & 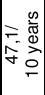 & & 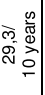 & & 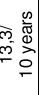 & & & & & & & & & & & & 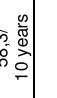 & & 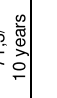 & . & & & & 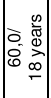 \\
\hline î & 总 & $\mid \begin{array}{l}\mathscr{m} \\
\stackrel{9}{\sigma}\end{array}$ & $\bar{o}$ & $\begin{array}{l}\text { 志 } \\
\infty\end{array}$ & $\mid \begin{array}{l}\infty \\
\infty \\
\infty\end{array}$ & $\mid \begin{array}{l}\mathscr{\infty} \\
\infty\end{array}$ & ঃ & $\frac{9}{6}$ & ్ֻరి & $\begin{array}{l}\mathscr{D} \\
\infty\end{array}$ & & $\ddot{m}_{m}$ & $\stackrel{0}{R}$ & $\frac{\infty}{\infty}$ & $\stackrel{N}{\curvearrowright}$ & 今̃ & 芯 & \begin{tabular}{l|l} 
\\
$\Xi$
\end{tabular} & 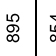 & 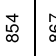 & 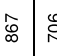 & 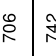 & 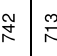 & $\stackrel{\infty}{\stackrel{\infty}{N}}$ & \begin{tabular}{l}
$\dot{1}$ \\
\hdashline \\
\hdashline
\end{tabular} & $\begin{array}{l}: \\
\stackrel{8}{f}\end{array}$ & న్ & 嵒 \\
\hline 穵 & $\begin{array}{l}\stackrel{\circ}{0} \\
\stackrel{0}{0} \\
\stackrel{\sim}{0}\end{array}$ & 总 & 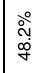 & $\begin{array}{l}\circ \\
\stackrel{\circ}{\circ} \\
\stackrel{\circ}{\circ}\end{array}$ & \begin{tabular}{|c|}
$\circ$ \\
$\stackrel{0}{0}$ \\
$\dot{5}$ \\
$\dot{\omega}$
\end{tabular} & $\begin{array}{l}\stackrel{\circ}{\grave{0}} \\
\hat{\theta}\end{array}$ & 总 & $\mid \frac{\circ}{\circ}$ & $\begin{array}{l}\circ \\
\vdots \\
\dot{j} \\
\dot{j}\end{array}$ & 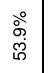 & & 空 & $\begin{array}{l}\circ \\
\circ \\
\infty \\
\infty \\
\infty\end{array}$ & $\begin{array}{c}\stackrel{\circ}{\stackrel{\circ}{े}} \\
\stackrel{\gamma}{*}\end{array}$ & $\begin{array}{l}\circ \\
\dot{\circ} \\
\dot{\infty} \\
\infty\end{array}$ & 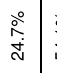 & : & 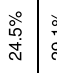 & $\frac{\circ}{\grave{ }}$ & $\begin{array}{l}\circ \\
\stackrel{\circ}{\circ} \\
\stackrel{\leftrightarrow}{\circ}\end{array}$ & $\begin{array}{ll}\circ \\
\stackrel{\circ}{\circ}\end{array}$ & 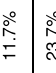 & 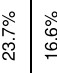 & 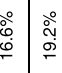 & & 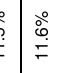 & $\begin{array}{l}\stackrel{0}{\circ} \\
\stackrel{0}{\mathrm{j}} \\
\stackrel{2}{2}\end{array}$ & $\begin{array}{l}\stackrel{\circ}{\circ} \\
\dot{ \pm}+\end{array}$ \\
\hline & $\begin{array}{l}\circ \\
\infty \\
\infty \\
\omega \\
\omega\end{array}$ & $\begin{array}{l}\stackrel{\circ}{0} \\
\stackrel{0}{\circ} \\
i\end{array}$ & $\begin{array}{l}\stackrel{\circ}{\circ} \\
\stackrel{\sigma}{N}\end{array}$ & $\frac{\stackrel{\circ}{\circ}}{\dot{g}}$ & \begin{tabular}{|c|}
$\circ$ \\
0 \\
0 \\
0
\end{tabular} & 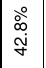 & $\begin{array}{l}\stackrel{\circ}{\circ} \\
\dot{m} \\
\dot{m}\end{array}$ & $\begin{array}{l}\stackrel{\circ}{\hat{2}} \\
\stackrel{\vec{f}}{*}\end{array}$ & $\begin{array}{l}\stackrel{\circ}{\grave{ }} \\
\stackrel{y}{\mathrm{~g}}\end{array}$ & 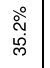 & 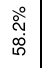 & \begin{tabular}{|c|}
$\stackrel{0}{0}$ \\
$\stackrel{0}{+}$
\end{tabular} & $\begin{array}{l}\stackrel{\circ}{\circ} \\
\dot{f} \\
\dot{m}\end{array}$ & $\mid \begin{array}{l}\grave{0} \\
\dot{0} \\
\dot{m}\end{array}$ & $\frac{\circ}{\stackrel{\circ}{\mathrm{j}}}$ & 㝵 & $\begin{array}{l}\stackrel{\circ}{\circ} \\
\stackrel{j}{+}\end{array}$ & \begin{tabular}{c|c}
$\stackrel{\circ}{\circ}$ \\
$\stackrel{+}{+}$
\end{tabular} & $\begin{array}{c}\stackrel{0}{\circ} \\
\stackrel{\infty}{\infty}\end{array}$ & $\begin{array}{c}\stackrel{\circ}{\circ} \\
\stackrel{\infty}{\infty}\end{array}$ & 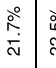 & 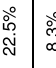 & \begin{tabular}{c|c}
$\circ$ & $\circ$ \\
$\infty$ & 0 \\
$\infty$ & 0 \\
\end{tabular} & 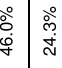 & & 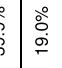 & \begin{tabular}{|l}
$\stackrel{\circ}{0}$ \\
$\dot{+}$ \\
$\dot{+}$
\end{tabular} & $\begin{array}{l}\circ \\
\stackrel{్}{~}\end{array}$ \\
\hline$\stackrel{\llcorner}{=}$ & $\stackrel{\mathscr{\rho}}{\stackrel{9}{\sigma}}$ & $\stackrel{\mathscr{N}}{\stackrel{2}{2}}$ & స̃ & $\underset{\sim}{\sim}$ & $\frac{m}{m}$ & : & 尺్లి & 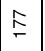 & 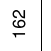 & 唐 & ल्ల & $\widehat{y}$ & $\underset{\sim}{\infty}$ & $\stackrel{\mathscr{B}}{\stackrel{8}{*}}$ & 总 & $\stackrel{\circ}{\circ}$ & ס̊ & $\bar{m}$ & ๙̃ ?ี & 今े & $\stackrel{\mathbb{\infty}}{\sim}$ & I & $\underset{F}{\exists}$ & 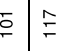 & $=\&$ & 8 & 怘 & $\approx$ \\
\hline & $\stackrel{\infty}{\infty}$ & $\hat{m}$ & के & $\stackrel{\square}{\circ}$ & \begin{tabular}{|l|}
$\stackrel{\infty}{\curvearrowright}$ \\
$\stackrel{2}{*}$
\end{tabular} & $\stackrel{\circ}{\circ}$ & $\stackrel{+}{N}$ & ळ & उ & $\overline{\mathrm{N}}$ & $\bar{\sigma}$ & ลี & $\underset{\infty}{\stackrel{\infty}{-}}$ & $\stackrel{\bullet}{\circ}$ & సે & 志 & $\stackrel{\infty}{\cong}$ & $\therefore$ & $\underset{\mathrm{I}}{\stackrel{\Delta}{ \pm}}$ & 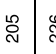 & $\stackrel{N}{*}$ & is & 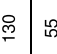 & \begin{tabular}{l|l} 
㟔 & Ф
\end{tabular} & $B$ & is & 요 & $\bar{\sigma}$ \\
\hline & is & $\stackrel{\mathscr{m}}{\stackrel{0}{*}}$ & $\Xi$ & 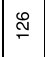 & $\stackrel{\circ}{\rightleftharpoons}$ & 总 & $\stackrel{\llcorner}{\rightleftharpoons}$ & ¿ & 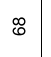 & $\stackrel{\circ}{\circ}$ & $\stackrel{\circ}{\circ}$ & 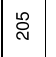 & $œ$ & む & $\therefore$ & م & $\cong$ & 芯 & 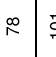 & 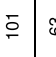 & 87 & $=\approx$ & $\cong F$ & $\begin{array}{l}f \\
\dot{f}\end{array}$ & $\stackrel{\infty}{\sim}$ & $=\simeq$ & is & $\bar{N}$ \\
\hline 兽 & 芯 & \begin{tabular}{|c}
$\stackrel{\circ}{\circ}$ \\
$\stackrel{\circ}{\circ}$
\end{tabular} & 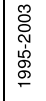 & 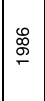 & \begin{tabular}{|l}
$\stackrel{\mathscr{S}}{\circ}$ \\
$\stackrel{8}{\circ}$
\end{tabular} & \begin{tabular}{|l}
$\stackrel{\infty}{\infty}$ \\
$\stackrel{2}{\sim}$
\end{tabular} & $\stackrel{\stackrel{\leftrightarrow}{\circ}}{\circ}$ & 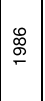 & $\stackrel{\mathscr{8}}{\stackrel{\leftrightarrow}{\circ}}$ & 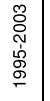 & 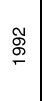 & 욤 & 兽 & 兽 & 兽 & 兽 & : & : & ঃั & 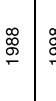 & $\begin{array}{l}\stackrel{\infty}{\circ} \\
\stackrel{8}{\circ}\end{array}$ & 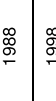 & 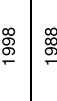 & 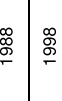 & 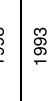 & 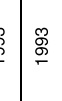 & 怘 & $\stackrel{N}{\stackrel{N}{N}}$ \\
\hline r & $\wedge$ & $\lambda$ & 우 & 0 & 0 & 0 & 0 & 0 & 0 & $\circ$ & $\wedge$ & 0 & 0 & $\infty$ & $\infty$ & $\infty$ & $\infty$ & $\infty$ & $\infty$ & $\infty \quad 0$ & $\infty$ & $\infty \quad \infty$ & $\infty \quad \infty$ & \begin{tabular}{l|l}
$\infty$ & $\infty$
\end{tabular} & \begin{tabular}{l|l}
0 & $\infty$
\end{tabular} & $\begin{array}{ll}\circ & \infty\end{array}$ & $\infty$ & $\wedge$ \\
\hline 号 & 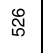 & & \&্ণ & 茖 & $\begin{array}{l}0 \\
i n \\
i n\end{array}$ & 品 & $\bar{i}$ & 亲 & ষ্ণ & 20 & & 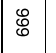 & 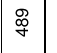 & 㟔 & $\stackrel{\circ}{\square}$ & $\stackrel{8}{8}$ & $\ddot{q}$ & 总 & 若 & $\begin{array}{l}\hat{f} \\
\dot{f}\end{array}$ & 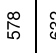 & ๗్ల & $8 \overline{0}$ & $\frac{\overline{0}}{6} \div$ & $\begin{array}{l}5 \\
5\end{array}$ & $\begin{array}{l}5 \\
b\end{array}$ & 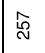 & ్ำ \\
\hline & & Os & & J & 足 & ○ & $\stackrel{\infty}{\infty}$ & ग) & J & & & 号 & 思 & \& & 足 & $\stackrel{0}{0}$ & $\stackrel{\mathscr{c}}{\mathrm{s}}$ & s) & 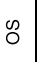 & & ?ु & & § & ב⿱ & $\begin{array}{l}3 \\
3\end{array}$ & $B$ & 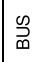 & \& \\
\hline 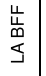 & $\begin{array}{l}\text { 岀 } \\
\text { 嵩 } \\
\text { s }\end{array}$ & 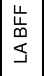 & \begin{tabular}{|l}
$\frac{u}{u}$ \\
盛
\end{tabular} & $\begin{array}{l}\text { 崖 } \\
\sum \\
\Sigma\end{array}$ & $\begin{array}{l}\frac{u}{u} \\
\frac{\omega}{\Sigma} \\
\end{array}$ & 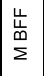 & $\begin{array}{l}\frac{u}{u} \\
\frac{m}{\Sigma} \\
\Sigma\end{array}$ & $\begin{array}{l}\frac{u}{u} \\
\stackrel{u}{m} \\
\Sigma\end{array}$ & $\begin{array}{l}\frac{u}{4} \\
\stackrel{\substack{\omega \\
\Sigma}}{2}\end{array}$ & 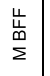 & \begin{tabular}{|l|} 
\\
$\frac{u}{\omega}$ \\
$\Sigma$ \\
$\Sigma$
\end{tabular} & \begin{tabular}{|l|l} 
\\
\\
$m$ \\
$\Sigma$ \\
$\Sigma$
\end{tabular} & 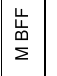 & \begin{tabular}{|l|l}
$\frac{u}{u}$ \\
\\
\end{tabular} & \begin{tabular}{|l|l}
$\frac{u}{\omega}$ \\
$\frac{m}{\Sigma}$
\end{tabular} & $\begin{array}{l}\frac{u}{\frac{u}{m}} \\
\frac{m}{\Sigma}\end{array}$ & $\begin{array}{l}\frac{u}{u} \\
\frac{m}{\Sigma}\end{array}$ & $\begin{array}{ll}\frac{u}{\omega} \\
\text { 盛 }\end{array}$ & \begin{tabular}{l|l}
$\frac{u}{u}$ \\
感
\end{tabular} & $\begin{array}{l}\frac{u}{\omega} \\
\stackrel{M}{\infty}\end{array}$ & \begin{tabular}{l|l}
$\frac{u}{u}$ \\
感
\end{tabular} & 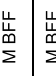 & 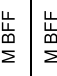 & \begin{tabular}{l|l}
$\frac{u}{u}$ & $\frac{u}{\omega}$ \\
$\Sigma$ & 盛
\end{tabular} & 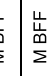 & 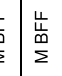 & 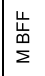 & \begin{tabular}{l|}
$\frac{u}{\omega}$ \\
\\
\end{tabular} \\
\hline 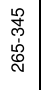 & 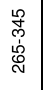 & 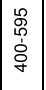 & 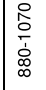 & ळ్ & 令 & 导 & 导 & 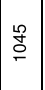 & 㝵 & 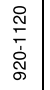 & 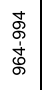 & 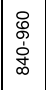 & 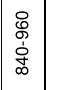 & \begin{tabular}{|c|}
0 \\
$\stackrel{0}{0}$
\end{tabular} & 号 & $\stackrel{\circ}{\circ}$ & జ్ & 足 & $\underset{\infty}{\infty}$ & 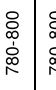 & 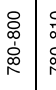 & 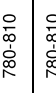 & 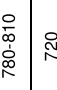 & \begin{tabular}{c|c} 
N \\
న
\end{tabular} & 응 & $\begin{array}{l}2 \\
2\end{array}$ & $\stackrel{\text { I }}{\stackrel{2}{ }}$ & 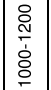 \\
\hline$\stackrel{\hat{\mathscr{\rho}}}{\circ}$ & $\stackrel{\widehat{\mathscr{S}}}{\circ}$ & 芯 & 惫 & 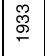 & \begin{tabular}{|l|}
$\stackrel{\infty}{\mathscr{J}}$ \\
$\stackrel{2}{\sim}$
\end{tabular} & 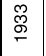 & 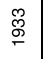 & 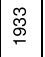 & $\underset{\mathscr{\varpi}}{\stackrel{\mathscr{m}}{\sigma}}$ & 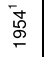 & $\begin{array}{l}\stackrel{+}{心} \\
\stackrel{\leftrightarrow}{\circ}\end{array}$ & & \begin{tabular}{|l|}
\multirow{J}{J}{} \\
$\stackrel{\sigma}{\circ}$
\end{tabular} & 㐫 & 皇 & S. & 兽 & 兽 & $\stackrel{s}{\stackrel{s}{\sigma}}$ & 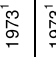 & 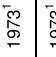 & 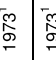 & 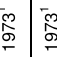 & 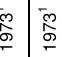 & $\begin{array}{l}\stackrel{5}{2} \\
\end{array}$ & 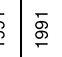 & 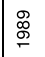 & 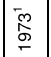 \\
\hline 03 & \% & is & is & 80 & 8 & 8 & 8 & 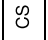 & 8 & 唨 & 8 & 83 & 8 & 8 & is & 8 & 8 & $\ddot{0}$ & 8 & o & $\mathscr{0}$ & 80 & \begin{tabular}{l|l}
8 & 0 \\
\end{tabular} & \begin{tabular}{l|l}
8 & 8
\end{tabular} & 3 & 30 & ช & के \\
\hline 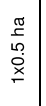 & 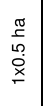 & 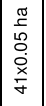 & 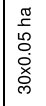 & 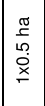 & 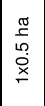 & 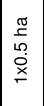 & 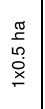 & 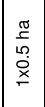 & 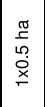 & 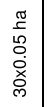 & \begin{tabular}{|l|}
$\underline{\sigma}$ \\
$\underline{\underline{x}}$ \\
$\underline{\underline{x}}$
\end{tabular} & 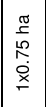 & 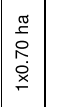 & 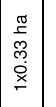 & \begin{tabular}{|l|}
$\underline{x}$ \\
$\underline{w}$ \\
$\tilde{p}$ \\
$\underline{p}$ \\
$\underline{\underline{x}}$ \\
\end{tabular} & 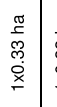 & 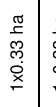 & 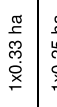 & 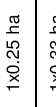 & 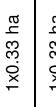 & 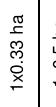 & 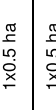 & 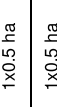 & 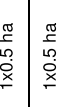 & & 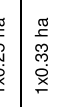 & 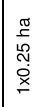 & 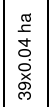 \\
\hline 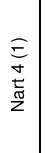 & $\begin{array}{l}\widehat{\widehat{N}} \\
\frac{+}{\tilde{N}} \\
\frac{\tilde{N}}{z}\end{array}$ & 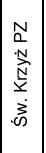 & 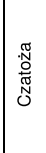 & 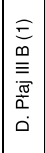 & 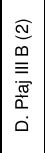 & 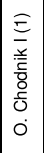 & $\begin{array}{l}\frac{\widehat{O}}{\bar{x}} \\
\frac{x}{\bar{z}} \\
\frac{\delta}{\partial} \\
0 \\
0\end{array}$ & 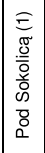 & 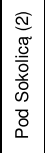 & 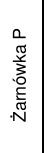 & 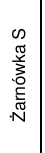 & 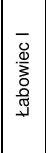 & 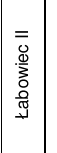 & \begin{tabular}{|l|}
$\bar{y}$ \\
$\tilde{J}$ \\
$\tilde{N}$ \\
$O$ \\
\end{tabular} & 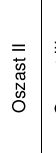 & 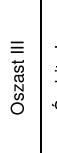 & 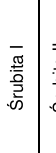 & 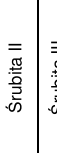 & 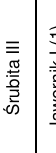 & 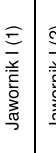 & 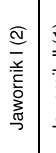 & 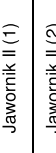 & 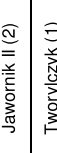 & 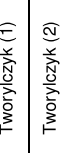 & 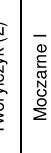 & 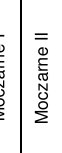 & 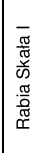 & 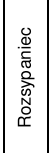 \\
\hline 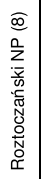 & 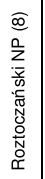 & 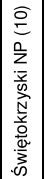 & 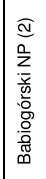 & 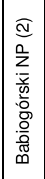 & 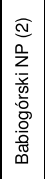 & 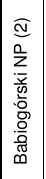 & 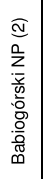 & 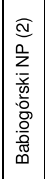 & 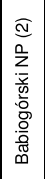 & 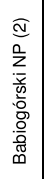 & 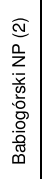 & 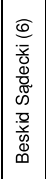 & 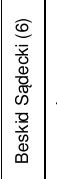 & 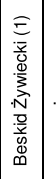 & 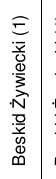 & 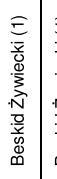 & 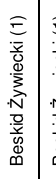 & 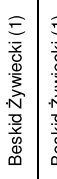 & 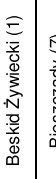 & 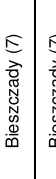 & 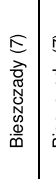 & 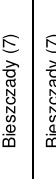 & 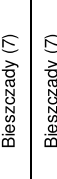 & 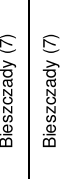 & 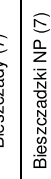 & 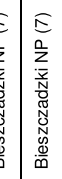 & 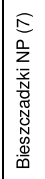 & 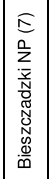 \\
\hline
\end{tabular}




\begin{tabular}{|c|c|c|c|c|c|c|c|c|c|c|c|c|c|c|c|c|c|c|c|c|c|c|c|c|c|c|c|c|}
\hline & 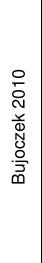 & 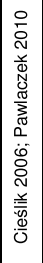 & & & 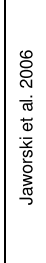 & 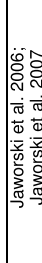 & & & & 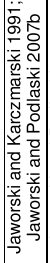 & & & & & & & & & & & & & & 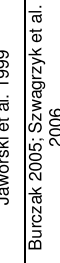 & 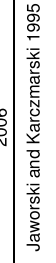 & & & \\
\hline & & & & & & & & & & & & & & & & & & & & & & & & & & |con & & \\
\hline$\widetilde{\sigma}$ & 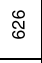 & 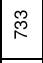 & 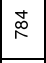 & 命 & $\begin{array}{c}\mathscr{R} \\
\stackrel{\infty}{\infty}\end{array}$ & 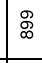 & $\stackrel{\varpi}{\infty}$ & 흠 & 売 & 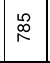 & $\stackrel{\widehat{\infty}}{\tilde{\infty}}$ & 总 & 草 & $\stackrel{8}{2}$ & $\bar{s}$ & $\bar{s}$ & $\frac{n}{6}$ & : & 吕 & : & $\widetilde{\Xi}$ & & 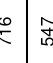 & $f$ & 总 & 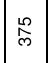 & 总 & 电 \\
\hline 彳̊ำ & 产 & 吕 & @̊․ & 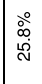 & 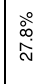 & 学 & 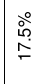 & 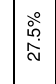 & 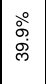 & $\begin{array}{l}\stackrel{\circ}{0} \\
\stackrel{\circ}{\circ}\end{array}$ & 离 & 吕 & 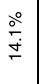 & 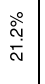 & مَّ & 宅 & 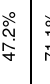 & 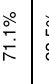 & 离 & @ & 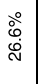 & & 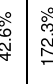 & 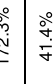 & 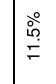 & $\begin{array}{c}\stackrel{\circ}{0} \\
\stackrel{\rho}{\rho} \\
\stackrel{2}{2}\end{array}$ & 突 & $\begin{array}{l}\stackrel{\circ}{\circ} \\
\stackrel{0}{\mathrm{~N}}\end{array}$ \\
\hline 空 & 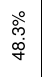 & 今ั & $\begin{array}{l}\circ \\
\vdots \\
\vdots \\
\vdots\end{array}$ & ๙̊ำ & 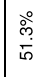 & ָَّْ & 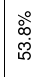 & 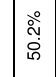 & \begin{tabular}{|l}
$\stackrel{\circ}{0}$ \\
$\stackrel{0}{0}$
\end{tabular} & $\begin{array}{l}\stackrel{\circ}{\circ} \\
\text { ơ }\end{array}$ & 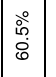 & 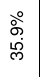 & 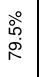 & ڤ̊ & $\begin{array}{l}\stackrel{8}{\circ} \\
\stackrel{\circ}{\circ}\end{array}$ & 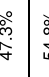 & 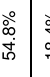 & 帣 & 突 & 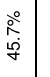 & 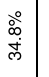 & & $\begin{array}{l}\circ \\
0 \\
0 \\
0\end{array}$ & $\frac{0}{\circ}$ & 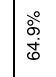 & $\begin{array}{l}\stackrel{\circ}{0} \\
\dot{0} \\
0 \\
0\end{array}$ & $\begin{array}{l}\text { ○ं } \\
\text { ơ }\end{array}$ & in \\
\hline 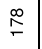 & $\stackrel{\mathbb{N}}{\text { N }}$ & $\stackrel{?}{?}$ & $\stackrel{?}{?}$ & $\stackrel{\leftrightarrow}{\circ}$ & $\Phi$ & ลิ & ల్ & 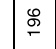 & $\stackrel{\infty}{\sim}$ & $\stackrel{\leftrightarrow}{\cong}$ & 它 & 刃ึ & $\bar{\infty}$ & $\stackrel{\mathbb{N}}{\underset{1}{2}}$ & 욤 & 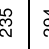 & 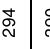 & : & 兽 & 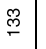 & $\stackrel{\infty}{=}$ & $\cong$ & 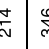 & 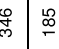 & ले & is & 产 & ১ \\
\hline$\cong$ & $\stackrel{\stackrel{\rho}{\rightleftarrows}}{=}$ & 8 & $\stackrel{\leftrightarrow}{\stackrel{\leftrightarrow}{\prime \prime}}$ & $\stackrel{\circ}{\underline{p}}$ & 8 & ¥ & $\bar{\sigma}$ & ळ & $\Phi$ & 8 & $\because$ & $\stackrel{q}{q}$ & 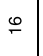 & $\stackrel{+}{\infty}$ & $\stackrel{ \pm}{\stackrel{2}{*}}$ & $\Xi$ & 商 & $\frac{9}{\mathrm{n}}$ & 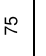 & $\approx$ & $\stackrel{\infty}{=}$ & & 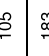 & 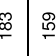 & $\stackrel{m}{m}$ & $\neq$ & 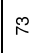 & 8 \\
\hline 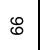 & 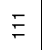 & $\infty$ & $\$$ & 另 & 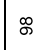 & 吕 & $\Sigma$ & \& & is & $\ddot{\circ}$ & 훙 & ¿ & ذे & o & เ & $F$ & $\bar{\Phi}$ & $\approx$ & \& & $\bar{\epsilon}$ & ஜ & 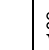 & 8 & $\stackrel{8}{:}$ & I & $\approx$ & : & f \\
\hline & ৯ & 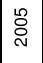 & $\stackrel{5}{\circ} \stackrel{2}{2}$ & ডà & & 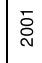 & & $\overline{\mathrm{a}}$ & 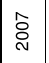 & 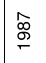 & 兽 & 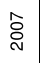 & 商 & 용 & & $\stackrel{\Phi}{\stackrel{\rho}{2}}$ & $\stackrel{s}{q}$ & ๙ & 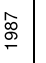 & 兽 & $\stackrel{\circ}{\circ}$ & ֻٌ & ఫ & 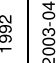 & 总 & $\stackrel{\circ}{\circ}$ & & \\
\hline
\end{tabular}

$\wedge$ ᄀ

手总兽

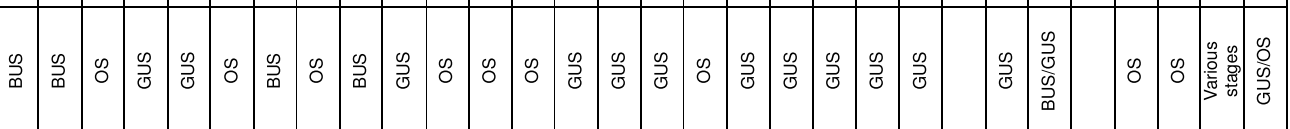

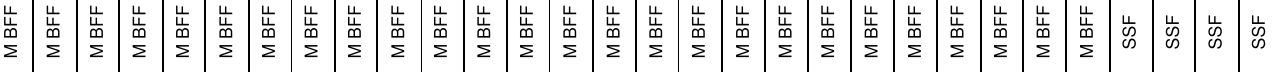

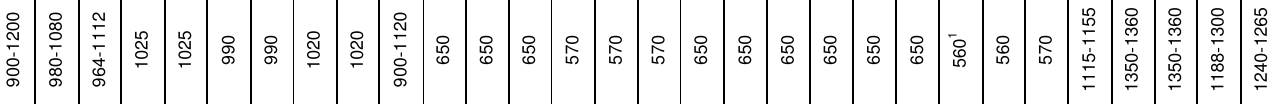

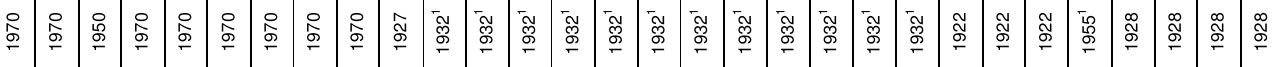

क क

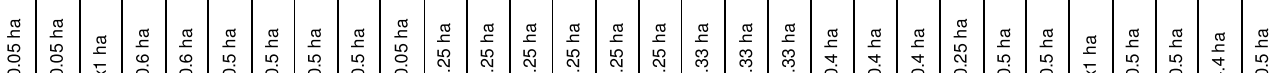

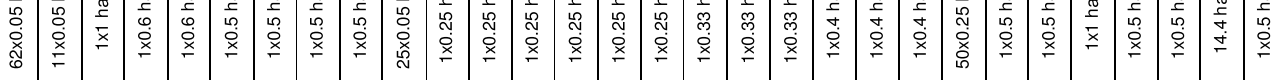

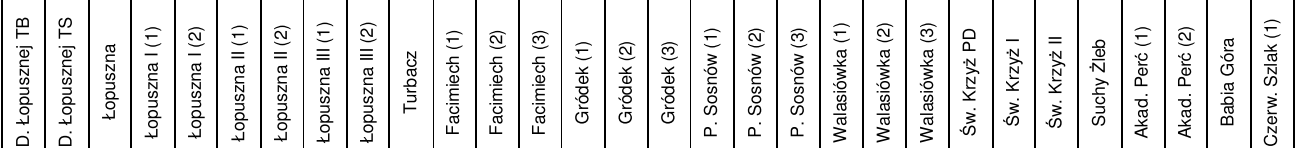




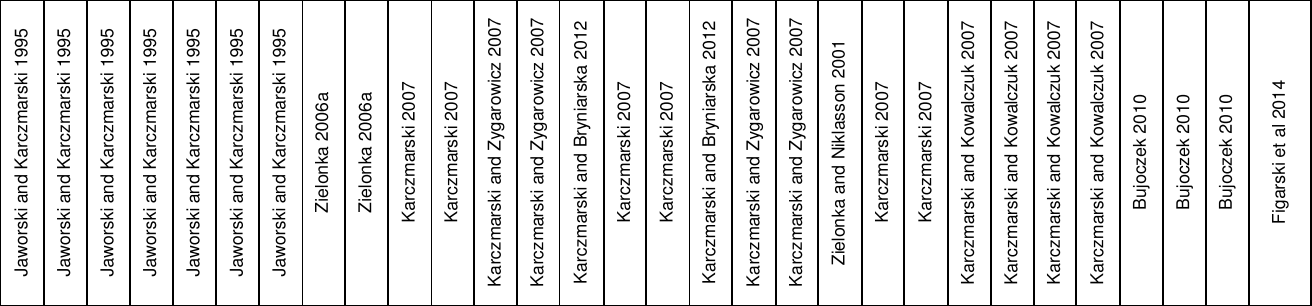

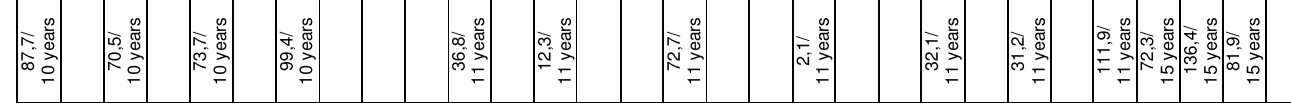

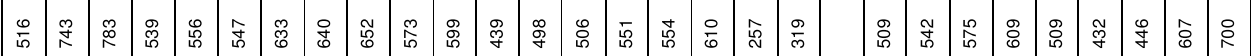

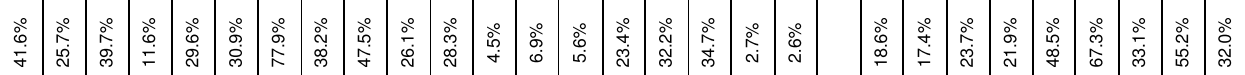

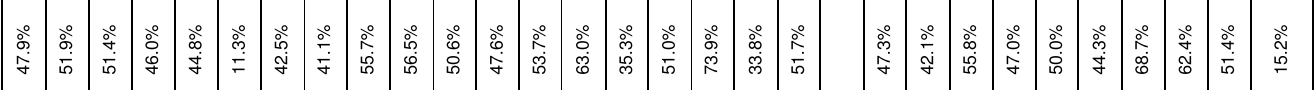

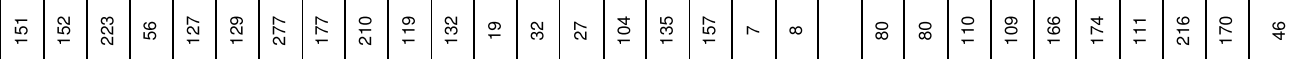

হ

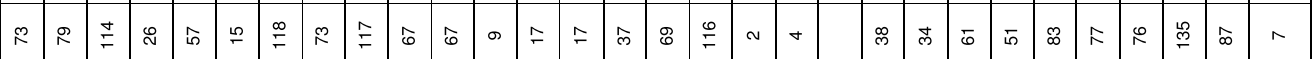

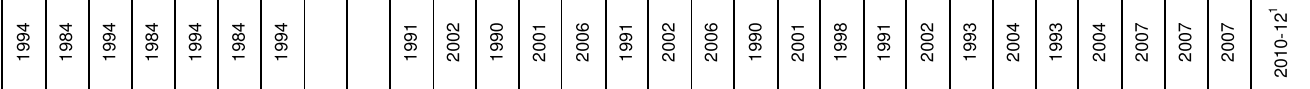

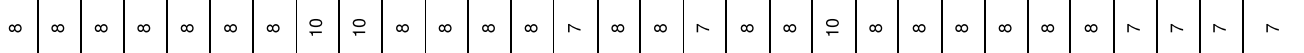

志畐蒠孚

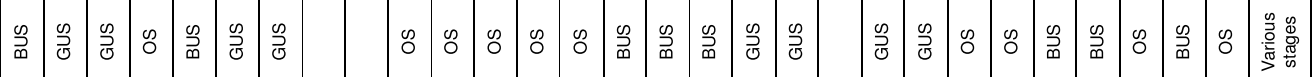

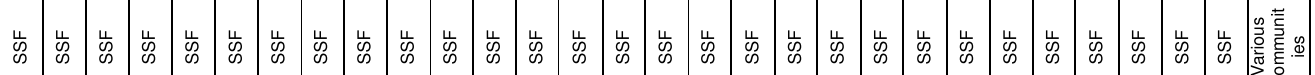

尊

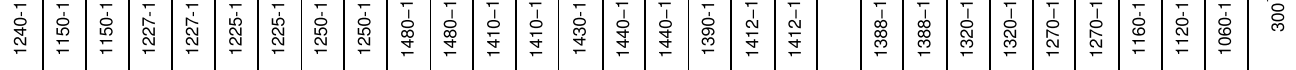

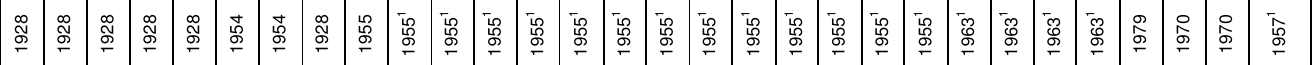

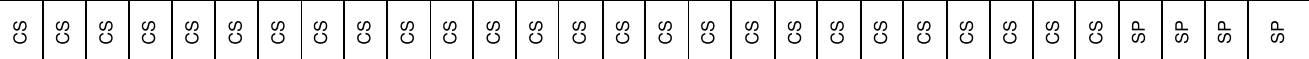

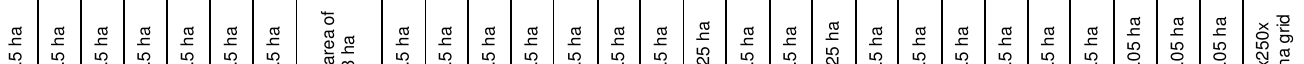

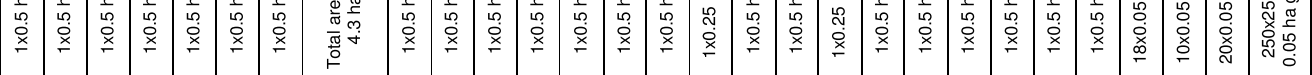

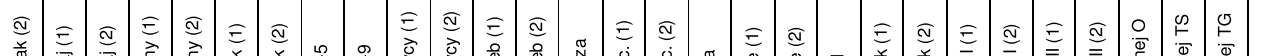

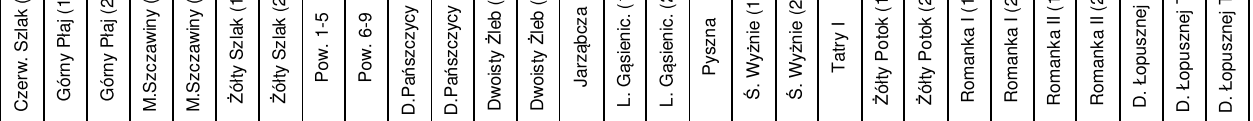

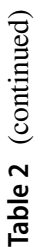
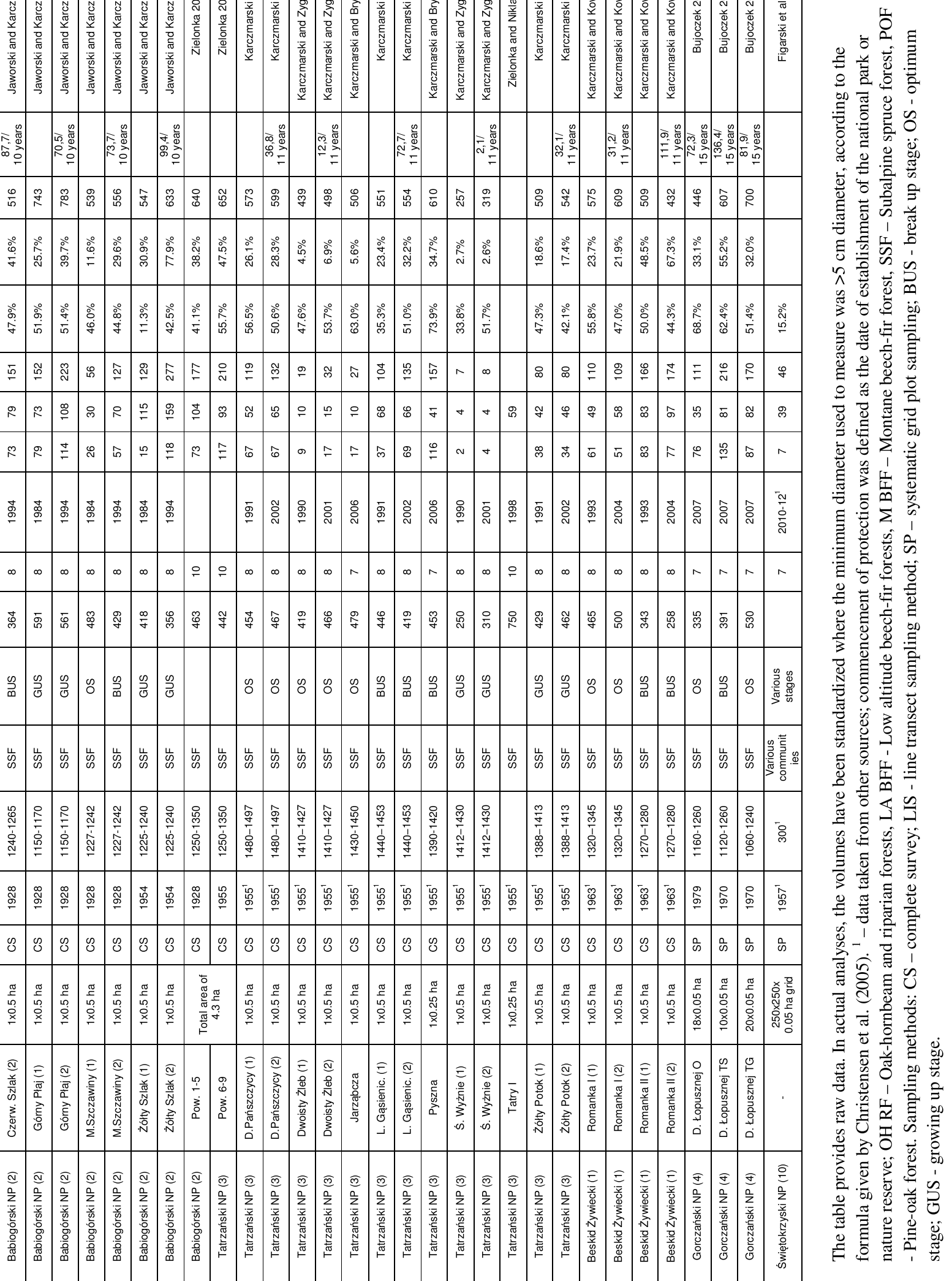


\section{References}

Bąk W (2012) Zasoby i struktura martwego drewna w drzewostanach objętych ochroną bierną w Ojcowskim Parku Narodowym. MSc thesis, University of Agriculture in Kraków

Banaś J, Bujoczek L, Zięba S, Drozd M (2014) The effects of different types of management, functions, and characteristics of stands in Polish forests on the amount of coarse woody debris. Eur J Forest Res 133:1095-1107. https://doi.org/10.1007/s10342-014-0825-3

Bobiec A (2002) Living stands and dead wood in the Bialowieża Forest: suggestions for restoration management. For Ecol Manag 165:125-140. https://doi.org/10.1016/S0378-1127(01)00655-7

Bouget C, Parmain G, Gilg O, Noblecourt T, Nusillard B, Paillet Y, Pernot C, Larrieu L, Gosselin F (2014) Does a set-aside conservation strategy help the restoration of old-growth forest attributes and recolonization by saproxylic beetles? Anim Conserv 17:342-353. https://doi.org/10.1111/acv.12101

Bujoczek L (2010) Wielkość, struktura i stopień rozkładu martwego drewna na tle zmian zasobów leśnych w rezerwatach Turbacz i Dolina Łopusznej. Ph.D. thesis. Department of Forest Management, Faculty of Forestry, University of Agriculture in Krakow

Bujoczek L, Bujoczek M (2016) Zasoby oraz zróżnicowanie martwego drewna w uroczysku Wapienny Las w Nadleśnictwie Polanów. Sylwan 160:482-491

Bujoczek L, Bujoczek M, Banaś J, Zięba S (2015) Spruce regeneration on woody microsites in a subalpine forest in the western Carpathians. Silva Fenn. https://doi.org/10.14214/sf.1337

Bujoczek L, Baraniewicz E, Banaś J, Zięba S (2017) Martwe drewno w buczynach objętych ochroną ścisłą uroczyska "Rozsypaniec" w Bieszczadzkim Parku Narodowym. Roczniki Bieszczadzkie 25:267-278

Burczak M (2004) Wpływ leżaniny na rozwój odnowienia buka, jodły i świerka na powierzchni badawczej "Suchy Żleb" w Tatrzańskim Parku Narodowym. MSc thesis, University of Agriculture in Kraków

Burrascano S, Lombardi F, Marchetti M (2008) Old-growth forest structure and deadwood: Are they indicators of plant species composition? A case study from central Italy. Plant Biosyst 142:313-323. https://doi.org/10.1080/11263500802150613

Bütler R, Angelstam P, Ekelund P, Schlaepfer R (2004) Dead wood threshold values for the three-toed woodpecker presence in boreal and sub-Alpine forest. Biol Conserv 119:305-318. https ://doi.org/10.1016/j.biocon.2003.11.014

Chećko E, Jaroszewicz B, Olejniczak K, Kwiatkowska-Falińska AJ (2015) The importance of coarse woody debris for vascular plants in temperate mixed deciduous forests. Can J For Res 45:1154-1163. https://doi.org/10.1139/cjfr-2014-0473

Christensen M, Hahn K, Mountford EP, Odor P, Standovar T, Rozenbergar D, Diaci J, Wijdeven S, Meyer P, Winter S, Vrska T (2005) Dead wood in European beech (Fagus sylvatica) forest reserves. For Ecol Manag 210:267-282. https://doi.org/10.1016/j.forec o.2005.02.032

Cieślik W (2006) Wpływ leżaniny na rozwój odnowienia buka, jodły i świerka na powierzchni badawczej "Łopuszna" w Gorczańskim Parku Narodowym. MSc thesis, University of Agriculture in Kraków

CSO (2014) Forestry. Central Statistical Office, Agriculture Statistics Division, Warsaw

Debeljak M (2006) Coarse woody debris in virgin and managed forest. Ecol Indic 6:733-742. https://doi.org/10.1016/j.ecoli nd.2005.08.031

Dittrich S, Jacob M, Bade C, Leuschner C, Hauck M (2014) The significance of deadwood for total bryophyte, lichen, and vascular plant diversity in an old-growth spruce forest. Plant Ecol 215:1123-1137. https://doi.org/10.1007/s11258-014-0371-6
Faliński JB (1978) Uprooted trees, their distribution and influence in the primeval forest biotope. Vegetatio 38:175-183. https://doi. org/10.1007/BF00123268

Figarski T, Buchholz L, Szczygielski M (2014) Struktura zasobów drewna martwych drzew w Świętokrzyskim Parku Narodowym i jego znaczenie dla zachowania populacji wybranych gatunków chrząszczy saproksylobiontycznych. Studia i Materiały CEPL 41:258-272

Franklin CMA, Harper KA, Murphy LK (2015) Structural dynamics at boreal forest edges created by a spruce budworm outbreak. Silva Fenn. https://doi.org/10.14214/sf.1267

Fukasawa Y, Katsumata S, Mori AS, Osono T, Takeda H (2014) Accumulation and decay dynamics of coarse woody debris in a Japanese old-growth subalpine coniferous forest. Ecol Res 29:257-269. https://doi.org/10.1007/s11284-013-1120-3

Gao T, Nielsen AB, Hedblom M (2015) Reviewing the strength of evidence of biodiversity indicators for forest ecosystems in Europe. Ecol Indic 57:420-434. https://doi.org/10.1016/j.ecoli nd.2015.05.028

Gutowski JM (2006) Saproksyliczne chrząszcze. Kosmos. Problemy Nauk Biologicznych 55:53-73

Herrmann S, Kahl T, Bauhus J (2015) Decomposition dynamics of coarse woody debris of three important central European tree species. For Ecosyst 2:1-14. https://doi.org/10.1186/s4066 3-015-0052-5

Hobi ML, Commarmot B, Bugmann H (2015) Pattern and process in the largest primeval beech forest of Europe (Ukrainian Carpathians). J Veg Sci 26:323-336. https://doi.org/10.1111/jvs.12234

Holeksa J (1998) Rozpad drzewostanu i odnowienie świerka a struktura i dynamika karpackiego boru górnoreglowego. Monographiae Botanicae 82

Holeksa J, Barć A, Hyla A, Krawczyk B (2006) Changes in coarse woody debris of a West Carpathian subalpine spruce forest over ten years. Polish Bot Stud 22:231-240

Holeksa J, Saniga M, Szwagrzyk J, Dziedzic T, Ferenc S, Wodka M (2007) Altitudinal variability of stand structure and regeneration in the subalpine spruce forests of the Pol'ana biosphere reserve, Central Slovakia. Eur J For Res 126:303-313. https:// doi.org/10.1007/s10342-006-0149-Z

Holeksa J, Zielonka T, Żywiec M (2008) Modeling the decay of coarse woody debris in a subalpine Norway spruce forest of the West Carpathians, Poland. Can J For Res 38:415-428. https://doi. org/10.1139/X07-139

Jamrozy G (2014) Ssaki polskich parków narodowych. Wydział Leśny Uniwersytetu Rolniczego, Kraków

Jaworski A, Jakubowska D (2011) Dynamika zmian budowy, struktury i składu gatunkowego drzewostanów o charakterze pierwotnym na wybranych powierzchniach w Pienińskim Parku Narodowym. Leśne Prace Badawcze 72:339-356. https://doi.org/10.2478/ v10111-011-0034-5

Jaworski A, Jakubowska D (2012) Ubytek, dorost i przyrost drzewostanów o charakterze pierwotnym na wybranych powierzchniach w Pienińskim Parku Narodowym. Sylwan 156:182-191

Jaworski A, Kaczmarski J (1990) Budowa i struktura drzewostanów dolnoreglowych o charakterze pierwotnym w Babiogórskim Parku Narodowym. Acta Agraria et Silvestria series Silvestris 29:49-63

Jaworski A, Karczmarski J (1991) Struktura i dynamika drzewostanów o charakterze pierwotnym w Pienińskim Parku Narodowym (na przykładzie czterech powierzchni doświadczalnych). Zeszyty Naukowe Akademii Rolniczej im H. Kołłątaja w Krakowie $254: 45-83$

Jaworski A, Karczmarski J (1995) Budowa, struktura, dynamika i możliwości produkcyjne górnoreglowych borów świerkowych w Babiogórskim Parku Narodowym. Acta Agraria et Silvestria series Silvestris 33:75-113 
Jaworski A, Kołodziej Z (2002) Natural loss of trees, recruitment and increment in stands of primeval character in selected areas of the Bieszczady Mountains National Park (South-Eastern Poland). J For Sci 48:141-149

Jaworski A, Paluch J (2001) Structure and dynamics of the lower mountain zone forest of primeval character in the Babia Góra Mt. National Park. J For Sci 47:60-74

Jaworski A, Paluch J (2002) Factors affecting the basal area increment of the primeval forests in the Babia Góra National Park, Southern Poland. Forstwissenschaftliches Centralblatt 121(3):97-108. https://doi.org/10.1046/j.1439-0337.2002.00097.x

Jaworski A, Podlaski R (2007a) Processes of loss, recruitment, and increment in stands of a primeval character in selected areas of the Pieniny National Park (Southern Poland). J For Sci 53:278-289

Jaworski A, Podlaski R (2007b) Structure and dynamics of selected stands of primeval character in the Pieniny National Park. Dendrobiology 58:25-42

Jaworski A, Skrzyszewski J, Świątkowski W, Kaczmarski J (1991) Budowa i struktura dolnoreglowych drzewostanów o charakterze pierwotnym na wybranych powierzchniach w Bieszczadach Zachodnich. Zeszyty Naukowe Akademii Rolniczej w Krakowie 254:17-43

Jaworski A, Karczmarski J, Skrzyszewski J (1994) Dynamika, budowa i struktura drzewostanów w rezerwacie "Łabowiec". Acta Agraria et Silvestria series Silvestris 32:3-26

Jaworski A, Pach M, Skrzyszewski J (1995) Budowa i struktura drzewostanów z udziałem buka i jawora w kompleksie leśnym Moczarne oraz pod Rabią Skałą (Bieszczady). Acta Agraria et Silvestria series Silvestris 33:39-73

Jaworski A, Podlaski R, Waga T (1999) Budowa i struktura drzewostanów o charakterze pierwotnym w rezerwacie Święty Krzyż [Świętokrzyski Park Narodowy]. Acta Agraria et Silvestria series Silvestris 37:27-51

Jaworski A, Kołodziej Z, Pach M (2001a) Skład gatunkowy, budowa i struktura drzewostanów w rezerwacie Śrubita. Sylwan 145:21-47

Jaworski A, Kołodziej Z, Strzęska T (2001b) Skład gatunkowy, budowa i struktura drzewostanów w rezerwacie Oszast. Sylwan 145:5-31

Jaworski A, Kołodziej Z, Porada K (2002) Structure and dynamics of stands of primeval character in selected areas of the Bieszczady National Park. J For Sci 48:185-201

Jaworski A, Kołodziej Z, Bartkowicz L (2005) Structure and dynamics of stands of primeval character composed of the little-leaf linden (Tilia cordata Mill.) in the "Las lipowy Obrożyska" reserve (southern Poland). J For Sci 51:283-304

Jaworski A, Kołodziej Z, Łapka M, Bartkowicz L (2006) Budowa, struktura i dynamika drzewostanów o charakterze pierwotnym w rezerwacie "Dolina Łopusznej" (Gorczański Park Narodowy). Leśne Prace Badawcze 4:35-59

Jaworski A, Kołodziej Z, Łapka M (2007) Mortality, recruitment, and increment of trees in the Fagus-Abies-Picea stands of a primeval character in the lower mountain zone. Dendrobiology 57:15-26

Kahl T, Arnstadt T, Baber K, Bässler C, Bauhus J, Borken W, Buscot F, Floren A, Heibl C, Hessenmöller D, Hofrichter M, Hoppe B, Kellner H, Krüger D, Linsenmair KE, Matzner E, Otto P, Purahong W, Seilwinder C, Schulze ED, Wende B, Weisser WW, Gossner MM (2017) Wood decay rates of 13 temperate tree species in relation to wood properties, enzyme activities and organismic diversities. For Ecol Manag 391:86-95. https://doi. org/10.1016/j.foreco.2017.02.012

Karczmarski J (2007) Budowa, struktura i dynamika górnoreglowych borów świerkowych o charakterze pierwotnym w dolinach Pańszczycy i Stawów Gąsienicowych (Tatrzański Park Narodowy) w okresie kontrolnym 1991-2002. Sylwan 11:41-59

Karczmarski J, Bąk M (2010) Skład gatunkowy, budowa i struktura oraz kierunki przemian drzewostanu $\mathrm{z}$ udziałem świerka $\mathrm{w}$ przygrzbietowej części rezerwatu Madohora w Bekidzie Małym. Leśne Prace Badawcze 71:369-380. https://doi.org/10.2478/ v10111-010-0032-z

Karczmarski J, Bryniarska C (2012) Budowa i struktura górnoreglowych borów świerkowych [Picea abies (L.) H. Karst] o charakterze pierwotnym w dolinach Jarząbczej i Pyszniańskiej (Tatrzański Park Narodowy). Leśne Prace Badawcze 73:45-55. https://doi. org/10.2478/v10111-012-0005-5

Karczmarski J, Kowalczuk P (2007) Budowa, struktura i dynamika górnoreglowego boru świerkowego o charakterze pierwotnym w rezerwacie Romanka w Beskidzie Żywieckim (w okresie kontrolnym 1993-2004). Acta Agraria et Silvestria series Silvestris 45:39-71

Karczmarski J, Kunz Ł (2010) Budowa i struktura naturalnego drzewostanu bukowego w rezerwacie "Zamkowa Góra" koło Kartuz. Leśne Prace Badawcze 71:239-248. https://doi.org/10.2478/ v10111-010-0020-3

Karczmarski J, Zygarowicz J (2007) Budowa, struktura i dynamika naturalnych górnoreglowych borów świerkowych w dolinie Rybiego Potoku (Tatrzański Park Narodowy) w okresie kontrolnym 1990-2001. Sylwan 12:3-20

Karjalainen L, Kuuluvainen T (2002) Amount and diversity of coarse woody debris within a boreal forest landscape dominated by Pinus sylvestris in Vienansalo wilderness, Eastern Fennoscandia. Silva Fenn 36:147-167

Korpel Š (1989) Pralesy Slovenska. Veda, Bratislava

Korpel Š (1995) Die Urwälder der Westkarpaten. G. Fischer, Stuttgart Krankina ON, Harmon ME (1995) Dynamics of the dead wood carbon pool in northwestern Russian boreal forest. Water Air Soil Pollut 82:227-238

Kuźnik E (2013) Procesy lasotwórcze w buczynie objętej ochroną bierną w uroczysku "Rozsypaniec" Bieszczadzkiego Parku Narodowego. Master's thesis. Department of Forest Management, Faculty of Forestry, University of Agriculture in Krakow

Larrieu L, Cabanettes A, Gonin P, Lachat T, Paillet Y, Winter S, Bouget C, Deconchat M (2014) Deadwood and tree microhabitat dynamics in unharvested temperate mountain mixed forests: a life-cycle approach to biodiversity monitoring. For Ecol Manag 334:163-173. https://doi.org/10.1016/j.foreco.2014.09.007

Lombardi F, Lasserre B, Tognetti R, Marchetti M (2008) Deadwood in relation to stand management and forest type in Central Apennines (Molise, Italy). Ecosystems 11:882-894. https://doi. org/10.1007/s10021-008-9167-7

Lombardi F, Lasserre B, Chirici G, Tognetti R, Marchetti M (2012) Deadwood occurrence and forest structure as indicators of oldgrowth forest conditions in Mediterranean mountainous ecosystems. Ecoscience 19:344-355. https://doi.org/10.2980/19-4-3506

Maciejewski Z (2006) Dynamika populacji gatunków drzewiastych w zbiorowiskach leśnych o różnej żyzności w Roztoczańskim Parku Narodowym. PhD thesis. Agricultural University in Kraków

Maciejewski Z, Szafraniec S (2014) Martwe drzewa w lasach naturalnych Roztoczańskiego Parku Narodowego i ich rola w zachowaniu populacji zagrożonych wyginięciem gatunków chrząszczy saproksylicznych. Studia i Materiały Centrum Edukacji Przyrodniczo-Leśnej 16(4):248-257

Maślak M, Orczewska A (2010) Zasoby martwego drewna w zbiorowisku kwaśnej buczyny niżowej leśnych obszarów chronionych Górnego Śląska. Studia i Materiały CEPL 2(25):368-376

Mataji A, Sagheb-Talebi K, Eshaghi-Rad J (2014) Deadwood assessment in different developmental stages of beech (Fagus orientalis Lipsky) stands in Caspian forest ecosystems. Int J Environ Sci Technol 11:1215-1222. https://doi.org/10.1007/s1376 2-014-0532-0

Merganičová K, Merganič J (2010) Coarse woody debris carbon stocks in natural spruce forests of Babia hora. J For Sci 56:397-405 
Motta R, Berretti R, Lingua E, Piussi P (2006) Coarse woody debris, forest structure and regeneration in the Valbona Forest Reserve, Paneveggio, Italian Alps. Forest Ecol Manag 235:155-163. https ://doi.org/10.1016/j.foreco.2006.08.007

Müller J, Bütler R (2010) A review of habitat thresholds for dead wood: a baseline for management recommendations in European forests. Eur J For Res 129:981-992. https://doi.org/10.1007/s1034 2-010-0400-5

Müller-Using S, Bartsch N (2009) Decay dynamic of coarse and fine woody debris of a beech (Fagus sylvatica $\mathrm{L}$.) forest in Central Germany. Eur J For Res 128:287-296. https://doi.org/10.1007/ s10342-009-0264-8

Paillet Y, Pernot C, Boulanger V, Debaive N, Fuhr M, Gilg O, Gosselin F (2015) Quantifying the recovery of old-growth attributes in forest reserves: a first reference for France. For Ecol Manag 346:51-64. https://doi.org/10.1016/j.foreco.2015.02.037

Pasierbek T (2007) Martwe drewno i dynamika jego rozkładu w dolnoreglowych lasach Beskidów Zachodnich. PhD thesis. Department of Ecology, W. Szafer Institute of Botany in Kraków, Polish Academy of Sciences

Pasierbek T, Holeksa J, Wilczek Z, Żywiec M (2007) Why the amount of dead wood in Polish forest reserves is so small? Nat Conserv 64:65-71

Pawicka K, Woziwoda B (2011) Bilans martwego drewna w rezerwacie "Polesie Konstantynowskie". Sylwan 155(12):851-858

Pawlaczek M (2010) Skład gatunkowy i struktura drzewostanu w rezerwacie Dolina Łopusznej w Gorczańskim Parku Narodowym. BSc thesis, University of Agriculture in Kraków

Pedlar JH, Pearce JL, Venier LA, McKenney DW (2002) Coarse woody debris in relation to disturbance and forest type in boreal Canada. For Ecol Manag 158:189-194. https://doi.org/10.1016/S0378 $-1127(00) 00711-8$

Petritan IC, Commarmot B, Hobi ML, Petritan AM, Bigler C, Abrudan IV, Rigling A (2015) Structural patterns of beech and silver fir suggest stability and resilience of the virgin forest Sinca in the Southern Carpathians, Romania. For Ecol Manag 356:184-195. https://doi.org/10.1016/j.foreco.2015.07.015

Podlaski R (2014) Martwe drewno w różnych stadiach i fazach rozwojowych lasu naturalnego. Studia i Materiały CEPL w Rogowie 41(4):151-158

Poznański R (1998) Dynamika zmian zasobow leśnych w rezerwacie "Święty Krzyż”. Rocznik Świętokrzyski Ser B - Nauki Przyrodnicze 25:1-14

Preikša Z, Brazaitis G, Marozas V, Jaroszewicz B (2015) Dead wood quality influences species diversity of rare cryptogams in temperate broadleaved forests. iForest 9:276-285. https://doi. org/10.3832/ifor1483-008

R Core Team (2017) R: a language and environment for statistical computing. R Foundation for Statistical Computing, Vienna, Austria. https://www.R-project.org/. Accessed 30 Nov 2017

Rock J, Badeck FW, Harmon ME (2008) Estimating decomposition rate constants for European tree species from literature sources. Eur J For Res 127:301-313. https://doi.org/10.1007/s1034 2-008-0206-x

Rondeux J, Sanchez C (2010) Review of indicators and field methods for monitoring biodiversity within national forest inventories. Core variable: Deadwood. Environ Monit Assess 164:617-630. https://doi.org/10.1007/s10661-009-0917-6
Seibold S, Bässler C, Baldrian P, Reinhard L, Thorn S, Ulyshen MD, Weiß I, Müller J (2016a) Dead-wood addition promotes nonsaproxylic epigeal arthropods but effects are mediated by canopy openness. Biol Conserv 204:181-188. https://doi.org/10.1016/j. biocon.2016.09.031

Seibold S, Bässler C, Brandl R, Büche B, Szallies A, Thorn S, Ulyshen MD, Müller J (2016b) Microclimate and habitat heterogeneity as the major drivers of beetle diversity in dead wood. J Appl Ecol 53:934-943. https://doi.org/10.1111/1365-2664.12607

Shorohova EV, Shorohov AA (2001) Coarse woody debris dynamics and stores in a boreal virgin spruce forest. Ecol Bull 49:129-135

Stachura-Skierczyńska K, Bobiec A (2008) Raport-Stare drzewa i martwe drewno w polskich lasach. OTOP, Warszawa

Stokland JN, Siitonen J, Jonsson BG (2012) Biodiversity in dead wood. Cambridge University Press, Cambridge

Svoboda M, Pouska V (2008) Structure of a Central-European mountain spruce old-growth forest with respect to historical development. For Ecol Manag 255:2177-2188. https://doi.org/10.1016/j. foreco.2007.12.031

Szewczyk J, Szwagrzyk J (1996) Tree regeneration on rotten wood and on soil in old-growth stand. Vegetatio 122:37-46. https://doi. org/10.1007/BF00052814

Szwagrzyk J, Sulowski W, Skrzydłowski T (2006) Struktura naturalnego drzewostanu buczyny karpackiej w Tatrach na tle naturalnych buczyn z innych masywów Karpat. Sylwan 150:3-15

Vacek S, Vacek Z, Bílek L, Hejcmanová P, Štícha V, Remeš J (2015) The dynamics and structure of dead wood in natural spruce-beech forest stand-a 40 year case study in the Krkonoše National Park. Dendrobiology 73:21-32. https://doi.org/10.12657/denbi 0.073 .003

Van Brusselen J (2011) Maintenance, conservation and appropriate enhancement of biological diversity in forest ecosystems. In: FOREST EUROPE, UNECE and FAO 2011: State of Europe's Forests 2011. Status and Trends in Sustainable Forest Management in Europe

Vandekerkhove K, De Keersmaeker L, Menke N, Meyer P, Verschelde P (2009) When nature takes over from man: Dead wood accumulation in previously managed oak and beech woodlands in Northwestern and Central Europe. For Ecol Manag 258:425-435. https ://doi.org/10.1016/j.foreco.2009.01.055

WISL (2014) Wielkoobszarowa inwentaryzacja stanu lasu. Wyniki za okres 2009-2013. Biuro Urządzania Lasu i Geodezji Leśnej. Sękocin Stary

Zhou L, Dal L, Gu H, Zhong L (2007) Review on the decomposition and influence factors of coarse woody debris in forest ecosystem. J For Res 18:48-54. https://doi.org/10.1007/s11676-007-0009-9

Zielonka T (2006) Quantity and decay stages of coarse woody debris in old-growth subalpine spruce forests of the western Carpathians, Poland. Can J For Res 36:2614-2622. https://doi.org/10.1139/ x06-149

Zielonka T (2006b) When does dead wood turn into a substrate for spruce replacement? J Veg Sci 17(6):739-746. https://doi. org/10.1111/j.1654-1103.2006.tb02497.x

Zielonka T, Niklasson M (2001) Dynamics of dead wood and regeneration pattern in natural spruce forest in the Tatra Mountains, Poland. Ecol Bull 49:159-163 